\title{
SERRIN'S OVER-DETERMINED PROBLEM ON RIEMANNIAN MANIFOLDS
}

\author{
MOUHAMED MOUSTAPHA FALL AND IGNACE ARISTIDE MINLEND
}

\begin{abstract}
Let $(\mathcal{M}, g)$ be a compact Riemannian manifold of dimension $N, N \geq 2$. In this paper, we prove that there exists a family of domains $\left(\Omega_{\varepsilon}\right)_{\varepsilon \in\left(0, \varepsilon_{0}\right)}$ and functions $u_{\varepsilon}$ such that

$$
\begin{cases}-\Delta_{g} u_{\varepsilon}=1 & \text { in } \Omega_{\varepsilon} \\ u_{\varepsilon}=0 & \text { on } \partial \Omega_{\varepsilon} \\ g\left(\nabla_{g} u_{\varepsilon}, \nu_{\varepsilon}\right)=-\frac{\varepsilon}{N} & \text { on } \partial \Omega_{\varepsilon}\end{cases}
$$

where $\nu_{\varepsilon}$ is the unit outer normal of $\partial \Omega_{\varepsilon}$. The domains $\Omega_{\varepsilon}$ are smooth perturbations of geodesic balls of radius $\varepsilon$. If, in addition, $p_{0}$ is a non-degenerate critical point of the scalar curvature of $g$ then, the family $\left(\partial \Omega_{\varepsilon}\right)_{\varepsilon \in\left(0, \varepsilon_{0}\right)}$ constitutes a smooth foliation of a neighborhood of $p_{0}$. By considering a family of domains $\Omega_{\varepsilon}$ in which (0.1) is satisfied, we also prove that if this family converges to some point $p_{0}$ in a suitable sense as $\varepsilon \rightarrow 0$, then $p_{0}$ is a critical point of the scalar curvature. A Taylor expansion of he energy rigidity for the torsion problem is also given.
\end{abstract}

2010 Mathematics Subject Classification. 58J05, 58J32,58J37, 35N10, 35N25 .

Keywords. Over-determined problem, foliation, isochoric profile.

\section{INTRODUCTION}

Let $(\mathcal{M}, g)$ be a compact Riemannian manifold of dimension $N \geq 2$. We are interested in this paper in the construction of smooth domains $\Omega \subset \mathcal{M}$ where there exists $u \in C^{2}(\bar{\Omega})$ such that

$$
\begin{cases}-\Delta_{g} u=1 & \text { in } \Omega \\ u=0 & \text { on } \partial \Omega \\ g\left(\nabla_{g} u, \nu\right)=-c . & \text { on } \partial \Omega,\end{cases}
$$

where $c$ is a positive constant, $\Delta_{g}=\operatorname{div}\left(\nabla_{g}\right)$ is the Laplace-Beltrami operator, and $\nu$ is the unit outer normal of $\partial \Omega$. In the Euclidean space (at least in $\mathbb{R}^{2}$ ), if a function $v$ satisfies the 
first two equations of (1.1) then the quantity

$$
\int_{\Omega}|\nabla v|^{2}
$$

is the torsional rigidity of the $\operatorname{rod} \Omega \times \mathbb{R}$. Namely the torque required for unit angle of twist per unit length. We refer to [25] for the precise derivation and its relation with bending a plane membrane and the motion of viscous fluids. Still in Euclidean space, it was proved by Serrin in [41] that a $C^{2}$ domain $\Omega$ in which (1.1) has a solution must be a ball. The argument of Serrin to prove his result relies on the moving plane method due to Alexandrov in [1]. In fact Alexandrov introduced the moving plane method while proving that an embedded constant mean curvature hyper-surface in $\mathbb{R}^{N}$ must be a sphere. Serrin's result can be also derived from the Alexandrov's. Namely if (1.1) has a solution then $\partial \Omega$ has constant mean curvatures, see the work of Farina-Kawohl in [20] and Choulli-Henrot [7].

While CMC hyper-manifolds are stationary sets for the area functional under volume preserving deformations, an over-determined problems arises when looking for a stationary set (under volume preserving deformations as well) to some energy functional given by some functional inequalities. In our case this energy is proportional to the inverse of the torsional rigidity:

$$
J(\Omega):=\inf \left\{\int_{\Omega}|\nabla u|_{g}^{2} \operatorname{dvol}_{g}: \int_{\Omega} u \operatorname{dvol}_{g}=1, u \in H_{0}^{1}(\Omega)\right\} .
$$

In particular minimizing $\Omega \mapsto J(\Omega)$ is equivalent to maximizing the torsion rigidity and therefore Serrin's result states that balls maximize the torsion rigidity as it can be also derived from the Faber-Krahn inequality, see for instance [6].

A smooth bounded domain $\Omega$ is stationary (or a critical point) for the functional $\Omega \mapsto J(\Omega)$ under volume preserving deformations if and only if there exists $u_{\Omega} \in H_{0}^{1}(\Omega)$ such that

$$
\begin{cases}-\Delta_{g} u_{\Omega}=J(\Omega) & \text { in } \Omega \\ u_{\Omega}=0 & \text { on } \partial \Omega \\ g\left(\nabla_{g} u_{\Omega}, \nu\right)=\text { Const. } & \text { on } \partial \Omega .\end{cases}
$$

We refer to Section 3 for more detailed explanations. In Euclidean space it is known from Serrin [41] and Weinberger's [44] work that stationary smooth domains are balls. In this paper, we will show that in a Riemannian manifold, geodesic balls can be perturbed to stationary sets for $J$. Before stating our result, let us recall some known results in the constructions of CMC hyper-manifolds. In [47], Ye proved that if $p_{0}$ is a non-degenerate critical point of the scalar curvature of $g$ then the geodesic ball $B_{\varepsilon}^{g}\left(p_{0}\right)$ might be perturbed to a CMC sub-manifolds with mean curvature equal $\frac{N-1}{\varepsilon}$, the mean curvature of Euclidean balls $B_{\varepsilon}(0)$ with radius $\varepsilon$. By taking advantages on the variational properties of the problem, Pacard and $\mathrm{Xu}$ showed in [37] that CMC hyper-manifolds with mean curvature $\frac{N-1}{\varepsilon}$ always exist and the number is not less than the category of $\mathcal{M}$. 
By direct computation, a solution to (1.1) in $\mathbb{R}^{N}$ is given by

$$
\phi_{0}^{\varepsilon}(x):=\frac{\varepsilon^{2}-|x|^{2}}{2 N}
$$

which clearly satisfies

$$
\begin{cases}-\Delta \phi_{0}^{\varepsilon}=1 & \text { in } B_{\varepsilon}(0) \\ \phi_{0}^{\varepsilon}=0 & \text { on } \partial B_{\varepsilon}(0) \\ \frac{\partial \phi_{0}^{\varepsilon}}{\partial \nu}=-\frac{\varepsilon}{N} & \text { on } \partial B_{\varepsilon}(0) .\end{cases}
$$

The main results in this paper parallel those of Ye and Pacard-Xu. We first prove the following:

Theorem 1.1. Let $(\mathcal{M}, g)$ be a compact Riemannian manifold of dimension $N \geq 2$. There exists $\varepsilon_{0}>0$ and a smooth function $\mathcal{F}: \mathcal{M} \times\left(0, \varepsilon_{0}\right) \longrightarrow \mathbb{R}$ such that for all $\varepsilon \in\left(0, \varepsilon_{0}\right)$, if $p$ is a critical point of the function $\mathcal{F}(., \varepsilon)$ then there exists a smooth domain $\Omega_{\varepsilon}$ and a function $u_{\varepsilon} \in C^{2}\left(\overline{\Omega_{\varepsilon}}\right)$ such that

$$
\begin{cases}-\Delta_{g} u_{\varepsilon}=1 & \text { in } \Omega_{\varepsilon} \\ u_{\varepsilon}=0 & \text { on } \partial \Omega_{\varepsilon} \\ g\left(\nabla_{g} u_{\varepsilon}, \nu_{\varepsilon}\right)=-\frac{\varepsilon}{N} & \text { on } \partial \Omega_{\varepsilon} .\end{cases}
$$

Here $\nu_{\varepsilon}$ stands for the unit outer normal of $\partial \Omega_{\varepsilon}$. Moreover we have

$$
\left\|\mathcal{F}(\cdot, \varepsilon)-S_{g}\right\|_{C^{2, \alpha}(\mathcal{M})} \leq C \varepsilon^{2},
$$

where $S_{g}$ is the scalar curvature of $\mathcal{M}$ and $C>0$ is a constant independent on $\varepsilon$.

Let us denote by $B_{\varepsilon}^{g}(p)$ the geodesic ball centered at $p$ with radius $\varepsilon$. The domains $\Omega_{\varepsilon}$ we construct are perturbations of geodesic balls in the sense that $\Omega_{\varepsilon}=\left(1+v^{p, \varepsilon}\right) B_{\varepsilon}^{g}(p)$, with $v^{p, \varepsilon}: \partial B_{\varepsilon}^{g}(p) \rightarrow \mathbb{R}$ satisfying

$$
\left\|v^{p, \varepsilon}\right\|_{C^{2, \alpha}\left(\partial B_{\varepsilon}^{g}(p)\right)} \leq c \varepsilon^{2}
$$

while the function $u_{\varepsilon}$ satisfies the estimates

$$
\left\|u_{\varepsilon}\right\|_{C^{2}\left(\overline{\Omega_{\varepsilon}}\right)} \leq c
$$

where the constant $c$ is independent on $\varepsilon$.

The fact that a solution to (1.5) exists is guaranteed by the existence of $\mathcal{F}$. Indeed the number of critical points of $p \mapsto \mathcal{F}(p, \varepsilon)$ is greater than the Lusternik-Shnirelman category of $\mathcal{M}$, see [3]. However (1.6) implies that near a topologically stable critical point of $S_{g}$, there exists a critical point of $\mathcal{F}(\cdot, \varepsilon)$. In particular if $p$ is a non-degenerate critical point of $S_{g}$ then the implicit function theorem implies that there exists a curve $p_{\varepsilon}$ of critical points of $\mathcal{F}(\cdot, \varepsilon)$. It is known from the work of Micheletti and Pistoia in [33] that for a generic metric on a manifold, all critical points of the scalar curvature are non-degenerate. This implies 
that for a generic metric $g^{\prime}$, a neighborhood of any critical point of $S_{g^{\prime}}$ can be foliated by CMC hyper-manifolds, similar to geodesic spheres, thanks to Ye's result. The analogous to this result is contained in the following

Theorem 1.2. Assume that $p_{0}$ is a non-degenerate critical point of the scalar curvature function $S_{g}$ of $(\mathcal{M}, g)$. Then, there exists $\varepsilon_{0}>0$ such that $\left(\partial \Omega_{\varepsilon}\right)_{\varepsilon \in\left(0, \varepsilon_{0}\right)}$ constitutes a smooth foliation of a neighborhood of $p_{0}$, where $\Omega_{\varepsilon}$ is a domain in which Serrin's over-determined problem (1.5) possesses a solution.

In fact we obtain a precise form of the boundary of the domains constructed in Theorem 1.2. Indeed, we proved that there exists a function $\omega^{\varepsilon}: S^{N-1} \rightarrow \mathbb{R}_{+}$such hat

$$
\partial \Omega_{\varepsilon}=\left\{\exp _{p_{0}}\left(\omega^{\varepsilon}(y) \sum_{i=1}^{N} y^{i} E_{i}\right), \quad y \in S^{N-1}\right\}
$$

and moreover the map $\varepsilon \mapsto \omega^{\varepsilon}$ satisfies $\left.\partial_{\varepsilon} \omega^{\varepsilon}\right|_{\varepsilon=0}=1$. In particular, we can see that the domains $\Omega_{\varepsilon} \subset B_{\delta_{\varepsilon}}^{g}\left(p_{0}\right)$, for some function $\delta_{\varepsilon}=\varepsilon+O\left(\varepsilon^{2}\right)$. Our next result can be merely seen as the converse of Theorem 1.2.

Theorem 1.3. Suppose that for every $\varepsilon>0$ there exist $\delta_{\varepsilon}>0$, a smooth domain $\Omega_{\varepsilon} \subset$ $B_{\delta_{\varepsilon}}^{g}\left(p_{0}\right)$ and a function $u_{\varepsilon} \in C^{2}\left(\overline{\Omega_{\varepsilon}}\right)$ such that

$$
\begin{cases}-\Delta_{g} u_{\varepsilon}=1 & \text { in } \Omega_{\varepsilon} \\ u_{\varepsilon}=0 & \text { on } \partial \Omega_{\varepsilon} \\ g\left(\nabla_{g} u_{\varepsilon}, \nu_{\varepsilon}\right)=-\frac{\varepsilon}{N} & \text { on } \partial \Omega_{\varepsilon} .\end{cases}
$$

Assume that

$$
\varepsilon^{-1}\left|\delta_{\varepsilon}-\varepsilon\right| \rightarrow 0 \quad \text { as } \varepsilon \rightarrow 0
$$

and

$$
\left\|u_{\varepsilon}\right\|_{C^{2}\left(\overline{\Omega_{\varepsilon}}\right)} \leq C,
$$

for some $C>0$ independent on $\varepsilon$. Then $p_{0}$ is a critical point of the scalar curvature $S_{g}$.

An other question of interest we study in this paper is the expansion of the isochoric profile corresponding to the torsion problem. We define the profile $\mathcal{T}_{\mathcal{M}}$ by

$$
\mathcal{T}_{\mathcal{M}}(v, g):=\inf _{|\Omega|_{g}=v} J(\Omega) .
$$

In particular, thanks to the Faber-Krahn inequality,

$$
\mathcal{T}_{\mathbb{R}^{N}}(v)=J\left(B_{1}\right)\left(\frac{\left|B_{1}\right|}{v}\right)^{-\frac{N+2}{N}} .
$$


Theorem 1.4. We have

$$
\mathcal{T}_{\mathcal{M}}(v, g)=\left(1-c_{N} v^{\frac{2}{N}} \max _{\mathcal{M}} S_{g}+O\left(v^{\frac{3}{N}}\right)\right) \mathcal{T}_{\mathbb{R}^{N}}(v),
$$

as $v \rightarrow 0$, where

$$
c_{N}=\frac{N+6}{6 N(N+4)}\left|B_{1}\right|^{-\frac{2}{N}} .
$$

This result suggests that torsion rigidity is maximized by sets located where scalar curvature is maximal. Let $g_{k}$ be a metric of constant sectional curvature $k$ on a manifold $\mathcal{M}^{\prime}$ with dimension $N$. Suppose that $\max _{\mathcal{M}} S_{g}<N(N-1) k$ then Theorem 1.4 implies that

$$
\mathcal{T}_{\mathcal{M}}(v, g)>\mathcal{T}_{\mathcal{M}^{\prime}}\left(v, g_{k}\right)=J\left(B_{v}^{g_{k}}\right), \quad \text { as } v \rightarrow 0,
$$

where $B_{v}^{g_{k}}$ is a geodesic ball ball with volume $v$ in $\left(\mathcal{M}, g_{k}\right)$. We quote [21] and [46] for some recent geometric comparisons of the energy torsional rigidity.

Remark 1.5. The result in this paper provides critical domains that concentrate at points. In a forthcoming work, we will be interested in concentrations at minimal submanifolds. Namely letting $K$ be a non-degenerate minimal submanifold of $\mathcal{M}$. Let $K_{\rho}$ be the geodesic neighborhood of $K$ with radius $\rho>0$. We will perturbe the tube $K_{\rho}$ to a domain $\Omega_{\rho}$ such that there exists a function $u_{\rho}$ which satisfies

$$
\begin{cases}-\Delta u_{\rho}=1 & \text { in } \Omega_{\rho} \\ u_{\rho}=0 & \text { on } \partial \Omega_{\rho} \\ g\left(\nabla_{g} u_{\rho}, \nu_{\rho}\right)=\text { Const. } & \text { on } \partial \Omega_{\rho} .\end{cases}
$$

In the CMC theory, minimal submanifolds play as well an important role. In comparison to $Y e$ 's result, nondegenracy of critical point of the scalar curvature is replaced by the fact $K$ is non-degenerate: the Jacobi operator about $K$ does not have zero eigenvalues. We might not expect existence for all $\rho>0$ small but a sequence of $\rho^{\prime} s$ will do. This is motivated by the work of Malchiodi and Montenegro in [31] and related works on CMC's concentrating along submanifolds, [18, 28, 29].

Construction of solutions to over-determined problems on Riemannian manifolds was first studied by Pacard and Sicbaldi in [36], where they study an over-determined problem for the first Dirichlet eignevalue $\lambda_{1}\left(\Omega_{\varepsilon}\right)$ :

$$
\begin{cases}-\Delta_{g} u_{\varepsilon}=\lambda_{1}\left(\Omega_{\varepsilon}\right) u_{\varepsilon} & \text { in } \Omega_{\varepsilon} \\ u_{\varepsilon}=0 & \text { on } \partial \Omega_{\varepsilon} \\ g\left(\nabla_{g} u_{\varepsilon}, \nu_{\varepsilon}\right)=\text { Const. } & \text { on } \partial \Omega_{\varepsilon} .\end{cases}
$$

Pacard and Sicbaldi in [36] proved that when the Riemannian manifolds has a non-degenerate critical point $p_{0}$ of the scalar curvature then it is possible to build extremal domains for any given volume small enough, and such domains are close to geodesic balls centered at $p_{0}$. 
This result has been improved by Delay and Sicbaldi [8] eliminating the hypothesis of nondegenerate critical point of the scalar curvature. In particular they showed the existence of extremal domain of small volume in any compact Riemannian manifold. Some other results and works on construction of solutions to over-determined problems on Riemannian manifolds can be found in $[8,9,34,39,42]$.

We shall now explain our argument of proof which is based on geometric variational arguments, see the work of [24], [14,16,17,37], for the construction of constant mean curvature hyper-surfaces and Delay-Sicbaldi [8], for the construction of extremal domains for the first eigenvalue of the Laplace-Beltrami operator. See also [2] and [3] for related abstract perturbative methods.

The idea is to perturbed a geodesic ball $B_{\varepsilon}^{g}(p)$. For any function $v \in L^{2}\left(S^{N-1}\right)$, we will consider the decomposition $v=v_{0}+\bar{v}$ where $\int_{S^{N-1}} \bar{v} \operatorname{dvol}_{S^{N-1}}=0$. We define the scaled metric $\bar{g}=\varepsilon^{-2} g$. For $\left(v_{0}, \bar{v}\right) \in \mathbb{R} \times C^{2, \alpha}\left(S^{N-1}\right)$ we consider the nearby sets of the rescaled ball $B_{\varepsilon}^{\bar{g}}(p)$ given by:

$$
B_{1+v}^{\bar{g}}(p):=\left\{\exp _{p}^{\bar{g}}\left(\left(1+v_{0}+\chi(x) \bar{v}(x /|x|)\right) \sum_{i=1}^{N} x^{i} E_{i}\right):|x|<1\right\},
$$

where $\chi$ is a radial cutoff function identically equal to 0 for $|x| \leq \frac{1}{4}$ and 1 for $|x| \geq \frac{1}{2}$.

The main idea is to find $p, v_{0}, \bar{v}$ such that Serrin's over-determined problem (1.1) is solvable. The first step consists in constructing a first approximate solution by solving only the Dirichlet problem: given a point $p \in \mathcal{M}$, there exists $\varepsilon_{0}>0$ such that for all $\varepsilon \in\left(0, \varepsilon_{0}\right)$ and for all $\left(v_{0}, \bar{v}\right) \in \mathbb{R} \times C^{2, \alpha}\left(S^{N-1}\right)$ satisfying

$$
\left|v_{0}\right| \leq \varepsilon_{0}, \quad\|\bar{v}\|_{C^{2, \alpha}\left(S^{n-1}\right)} \leq \varepsilon_{0} \quad \text { and } \quad \int_{S^{N-1}} \bar{v} \mathrm{dvol}_{S^{N-1}}=0,
$$

there exists a unique positive function $\bar{\phi}=\bar{\phi}\left(p, \varepsilon, v_{0}, \bar{v}\right) \in C^{2, \alpha}\left(B_{1+v}^{\bar{g}}(p)\right)$ such that

$$
\begin{cases}-\Delta_{\bar{g}} \bar{\phi}=1 & \text { in } B_{1+v}^{\bar{g}}(p) \\ \bar{\phi}=0 & \text { on } \partial B_{1+v}^{\bar{g}}(p),\end{cases}
$$

This is done removing the dependence of the domains on the parameters by considering a change of variable via the function $Y_{p, v}$

$$
Y_{p, v}(x):=\exp _{p}^{\bar{g}}\left(\left(1+v_{0}+\chi(x) \bar{v}(x /|x|)\right) \sum_{i=1}^{N} x^{i} E_{i}\right)
$$

which parameterizes $B_{1+v}^{\bar{g}}(p)$ over the unit ball $B_{1}$ centered at the origin. Hence with the pull-back metric $\hat{g}$ of $\bar{g}$ with respect to $Y_{\varepsilon, v},(1.12)$ becomes

$$
\begin{cases}-\Delta_{\hat{g}} \hat{\phi}=1 & \text { in } B_{1} \\ \hat{\phi}=0 & \text { on } \partial B_{1} .\end{cases}
$$


Once we find $v_{0}, \bar{v}$ so that (1.13) holds for all $p \in \mathcal{M}$, we compute the normal derivative of $\hat{\phi}$. Denote by $\hat{\nu}$ the unit outward normal to $B_{1}$ with respect to the metric $\hat{g}$. We have obtained

$$
\hat{g}\left(\nabla_{\hat{g}} \hat{\phi}, \hat{\nu}\right)_{\left.\right|_{\partial B_{1}}}=-\left|\nabla_{g} \hat{\phi}\right|_{\hat{g}}=-\frac{1}{N}+\frac{1}{N}\left[\left(\partial_{\nu} \psi_{v}\right)_{\left.\right|_{\partial B_{1}}}-v\right]+\text { error },
$$

where $\psi_{v}$ satisfies

$$
\begin{cases}\Delta \psi_{\bar{v}}=0 & \text { in } B_{1} \\ \psi_{\bar{v}}=\bar{v} & \text { on } \partial B_{1} .\end{cases}
$$

The second step is to find $p, v_{0}, \bar{v}$ such that

$$
G\left(\varepsilon, p, v_{0}, \bar{v}\right):=\frac{1}{N}\left[\left(\partial_{\nu} \psi_{v}\right)_{\mid \partial B_{1}}-v\right]+\text { error }=0 .
$$

Direct computations then give

$$
\frac{\partial G}{\partial\left(v_{0}, \bar{v}\right)}(0, p, 0,0)\left[w_{0}, \bar{w}\right]=\frac{1}{N}\left[\left(\partial_{\nu} \psi_{w}\right)_{\left.\right|_{\partial B_{1}}}-w\right] .
$$

Note that the map $\bar{v} \mapsto \partial_{\nu} \psi_{\left.\bar{v}\right|_{\partial B_{1}}}$ is the classical Dirichlet-to-Neuman operator. Its spectrum is known and the eigenvalues are the spherical harmonics. It is then easy to verify that

$$
\operatorname{Ker} \frac{\partial G}{\partial\left(v_{0}, \bar{v}\right)}(0, p, 0,0)=\left\{x^{i}: i=1, \ldots, N\right\} .
$$

This allows us to solve (1.15) modulo its kernel. Namely there exist $v^{\varepsilon, p}=v_{0}^{\varepsilon, p}+\left\langle a^{\varepsilon, p}, x\right\rangle+$ $\bar{v}^{\varepsilon, p}$ such that

$$
G\left(\varepsilon, p, v_{0}^{\varepsilon, p}, \bar{v}\right)=-\left\langle a^{\varepsilon, p}, x\right\rangle, \quad \forall x \text { in } S^{N-1} .
$$

Gathering what we have so far, we may say that we have found a function $\bar{\phi}^{\varepsilon, p}$ satisfying

$$
\begin{cases}-\Delta_{\bar{g}} \bar{\phi}^{\varepsilon, p}=1 & \text { in } B_{1+v^{\varepsilon, p}}^{\bar{g}}(p) \\ \bar{\phi}^{\varepsilon, p}=0 & \text { on } \partial B_{1+v^{\varepsilon, p}}^{\bar{g}}(p) \\ \bar{g}\left(\nabla_{\bar{g}} \bar{\phi}^{\varepsilon, p}, \bar{\nu}\right)=-\frac{1}{N}-\bar{g}\left(A^{\varepsilon, p}, \mathcal{V}^{\varepsilon, p}\right) & \text { on } \partial B_{1+v^{\varepsilon, p}}^{\bar{g}}(p),\end{cases}
$$

where for all $x \in S^{N-1}$, we define $A^{\varepsilon, p}\left(Y_{p, v^{\varepsilon, p}}(x)\right):=d Y_{p, v^{\varepsilon, p}}(x)\left[a^{\varepsilon, p}\right]$ and similarly $\mathcal{V}^{\varepsilon, p}:=$ $d Y_{p, v^{\varepsilon, p}}(x)[x]$. This program is detailed in Paragraph 3.1.

Let us remind that the domains we are looking for are critical points of the energy functional $J(\Omega)$ under volume constraints and thus by the Lagrange multiplier rule, they are critical points of $J(\Omega)+\lambda|\Omega|_{\bar{g}}$, for some $\lambda \in \mathbb{R}$. We will take this advantage in the third step to annihilate $A^{\varepsilon, p}$ for some special points $p$. Indeed by defining

$$
\Phi_{\varepsilon}(p)=J\left(B_{1+v^{\varepsilon, p}}^{\bar{g}}(p)\right)+\frac{1}{N^{2}}\left|B_{1+v^{\varepsilon, p}}^{\bar{g}}(p)\right|_{\bar{g}},
$$


we will show that if $p$ is a critical points of this functional then $A^{\varepsilon, p}=0$. Rescaling back, we get the desired result: there exists a function $u_{\varepsilon}$ such that

$$
\begin{cases}-\Delta_{g} u_{\varepsilon}=1 & \text { in } B_{\varepsilon\left(1+v^{\varepsilon, p}\right)}^{g}(p) \\ u^{\varepsilon}=0 & \text { on } \partial B_{\varepsilon\left(1+v^{\varepsilon, p}\right)}^{g}(p) \\ g\left(\nabla_{g} u^{\varepsilon}, \nu_{\varepsilon}\right)=-\frac{\varepsilon}{N} & \text { on } \partial B_{\varepsilon\left(1+v^{\varepsilon, p}\right)}^{g}(p) .\end{cases}
$$

We refer to Paragraph 3.2 for more details. In addition the functional $\Phi_{\varepsilon}$ has a Taylor expansion for which the main term is given by the scalar curvature, see Lemma 4.1.

Next, in Section 5, we will prove that we have a smooth foliation near non-degenerate critical points of the scalar curvature. Here we take advantages of the expansion of $\Phi_{\varepsilon}(p)=$ $\alpha_{n}+\beta_{n} \varepsilon^{2} S_{g}(p)+O\left(\varepsilon^{4}\right)$ to see that provided $p_{0}$ is a non-degenerate critical point of the scalar curvature there exists a curve $p_{\varepsilon}$ of critical points of $\Phi_{\varepsilon}$ such that $\operatorname{dist}_{g}\left(p_{\varepsilon}, p_{0}\right) \leq C \varepsilon^{2}$. This fact allows us to re-parameterize $\partial B_{\varepsilon\left(1+v^{\varepsilon, p}\right)}^{g}(p)$ by perturbed sphere with increasing radius $\omega^{\varepsilon}$. Indeed there exists a nonnegative function $\omega^{\varepsilon}$ such that

$$
\partial B_{\varepsilon\left(1+v^{\left.\varepsilon, p_{\varepsilon}\right)}\right.}^{g}\left(p_{\varepsilon}\right)=\left\{\exp _{p_{0}}\left(\omega^{\varepsilon}(y) y^{i} E_{i}\right): y \in S^{N-1}\right\},
$$

with

$$
\omega^{\varepsilon}(\cdot)>0, \quad \text { and } \quad \partial_{\varepsilon} \omega^{\varepsilon}(\cdot)>0
$$

It is worth noticing that from our argument to prove local foliation, the sets $\Omega_{\varepsilon}$ in (1.11) constructed by Pacard and Sicbaldi in [36] enjoys such a local foliation, see Remark 5.2.

Finally in Section 6, we prove Theorem 1.3. The proof is based on the regularity result of nearly minimizing sets for the perimeter functional. Indeed, just integrating the (1.8), we see that the domains $\Omega_{\varepsilon}$ satisfies

$$
\left|\partial \Omega_{\varepsilon}\right|_{g}=\frac{N}{\varepsilon}\left|\Omega_{\varepsilon}\right|_{g}
$$

while (1.9) shows that they are contained in the ball $(\varepsilon+o(\varepsilon)) B_{1}$. This implies that

$$
\left|\partial \Omega_{\varepsilon}\right|_{g} \leq(1+o(1)) c_{N}\left|\Omega_{\varepsilon}\right|_{g}^{\frac{N-1}{N}}
$$

where $c_{N}=N|B|^{\frac{1}{N}}$ is the isoperimetric constant of $\mathbb{R}^{N}$. Therefore up to a scaling they nearly minimize the area functional among domains with volume $\left|B_{1}\right|$. Using some simple arguments, we deduce that they have bounded boundary mean curvatures. This leads to smooth convergence to the unit ball. We note that even if our argument works also when considering CMCs instead of critical domains, we choose not to expose it here. Among others we quote [12], [35], [43], [26] and [29], where the authors characterized the sets where a sequence of CMC's hyper-surfaces converges as their mean curvature tends to infinity. From the work of [43] and [26], it is also naturel to expect that the assumption (1.9) can be relaxed. 
Acknowledgements: This work is supported by the Alexander von Humboldt foundation and the German Academic Exchange Service (DAAD).

\section{Preliminaries AND notations}

Given a point $p \in \mathcal{M}$, we let $E_{1}, \ldots, E_{N}$ be an orthonormal basis of the tangent plane $T_{p} \mathcal{M}$. We consider geodesic coordinate system

$$
\mathbb{R}^{n} \ni\left(x^{1}, \ldots, x^{N}\right)=x \longmapsto F_{p}(x):=\exp _{p}(X) \in \mathcal{M},
$$

where we use here and in the following the notation

$$
X:=\sum_{i=1}^{N} x^{i} E_{i} \in T_{p} \mathcal{M}
$$

The map $F_{p}$ induces coordinate vector fields

$$
X_{i}:=d F_{p}(x)\left[E_{i}\right]
$$

We denote by

$$
R_{p}: T_{p} \mathcal{M} \times T_{p} \mathcal{M} \times T_{p} \mathcal{M} \longrightarrow T_{p} \mathcal{M}
$$

the Riemanniann curvature tensor at $p$ and

$$
\left.\operatorname{Ric}_{p}: T_{p} \mathcal{M} \times T_{p} \mathcal{M} \longrightarrow \mathbb{R}, \quad \operatorname{Ric}_{p}(X, Y)=-\sum_{i=1}^{N} g\left(R_{p}\left(X, E_{i}\right) Y, E_{i}\right)\right)
$$

the Ricci curvature tensor at $p$. The scalar curvature of $(\mathcal{M}, g)$ at $p$ is defined by

$$
S_{g}(p)=\sum_{k=1}^{N} \operatorname{Ric}_{p}\left(E_{k}, E_{k}\right)
$$

At a point $q=\exp _{p}(X)$, we define

$$
g_{i j}(x):=g\left(X_{i}, X_{j}\right) .
$$

The proof of the expansion of the metric $g$ near $p$ in normal coordinates is classical and can be found in [45] or [40].

Proposition 2.1. At a point $q=\exp _{p}(X)$, we have

$$
g_{i j}(x)=\delta_{i j}+\frac{1}{3} g\left(R_{p}\left(E_{i}, X,\right) E_{j}, X\right)+\frac{1}{6} g\left(\nabla_{X} R_{p}\left(E_{i}, X\right) E_{j}, X\right)+O_{p}\left(|x|^{4}\right),
$$

as $|x| \rightarrow 0$.

Let $f: S^{N-1} \longrightarrow(0, \infty)$ be a continuous function whose $L^{\infty}$ norm is small (say less than the cut locus of $p$ ). We can decompose $f$ into $f=f_{0}+\bar{f}$, where $f_{0}$ is a constant and $\bar{f}$ has mean value equal to 0 . We define

$$
B_{f}^{g}(p):=\left\{\exp _{p}^{g}\left(\left(f_{0}+\chi \bar{f}(x /|x|)\right) X\right) \quad: \quad|x|<1\right\}
$$


where $\chi$ is a radial cutoff function identically equal to 0 for $|x| \leq \frac{1}{4}$ and 1 for $|x| \geq \frac{1}{2}$. In particular if $f_{0} \equiv r$ a positive constant and $\bar{f}=0$, then $B_{f}^{g}(p)$ is nothing but the geodesic ball centered at $p$ with radius $r$.

Similarly, we denote by $|\Omega|_{g}$ the volume in the metric $g$ of a smooth domain $\Omega \subset \mathcal{M}$, $\mathrm{dvol}_{g}$ the volume element in the metric $g$ to integrate over the domain and $d \sigma_{g}$ denotes the volume element in the induce metric $g$ to integrate over the boundary of a domain. $\Delta_{g}$ and $\nabla_{g}$ denotes respectively, the Laplace-Beltrami and the gradient operator with respect to the metric $g$. It will be understood that when we do not indicate the metric as a superscript, we understand that we are using the Euclidian one.

Our aim is to show that, for $\varepsilon>0$ small enough, we can find a point $p$ and a (small) function $v: S^{N-1} \longrightarrow(0, \infty)$ such that, on $(\mathcal{M}, g)$, the over-determined problem

$$
\begin{cases}-\Delta_{g} u=1 & \text { in } B_{\varepsilon(1+v)}^{g}(p) \\ u=0 & \text { on } \partial B_{\varepsilon(1+v)}^{g}(p) \\ g\left(\nabla_{g} u, \nu\right)=-\frac{\varepsilon}{N} & \text { on } \partial B_{\varepsilon(1+v)}^{g}(p)\end{cases}
$$

has a solution, where $\nu$ is the unit outer normal vector about $\partial B_{\varepsilon(1+v)}^{g}(p)$.

We consider the dilated metric $\bar{g}=\varepsilon^{-2} g$ and rewrite $(2.1)$ on $(\mathcal{M}, \bar{g})$, as

$$
\begin{cases}-\Delta_{g} \bar{u}=1 & \text { in } B_{1+v}^{\bar{g}}(p) \\ \bar{u}=0 & \text { on } \partial B_{1+v}^{\bar{g}}(p) \\ \bar{g}\left(\nabla_{\bar{g}} \bar{u}, \bar{\nu}\right)=-\frac{1}{N} & \text { on } \partial B_{1+v}^{\bar{g}}(p),\end{cases}
$$

where

$$
\bar{u}=\varepsilon^{-2} u .
$$

The Taylor expansion of the scaled metric $\bar{g}$ can be easily derived from Proposition 2.1. Indeed we have

$$
\bar{g}_{i j}(x)=g_{i j}(\varepsilon x)=\delta_{i j}+\frac{\varepsilon^{2}}{3} g\left(R_{p}\left(E_{i}, X\right) E_{j}, X\right)+\frac{\varepsilon^{3}}{6} g\left(\nabla_{X} R_{p}\left(E_{i}, X\right) E_{j}, X\right)+O_{p}\left(\varepsilon^{4}\right) .
$$

Given $v \in C^{2, \alpha}\left(S^{N-1}\right)$, with $\alpha \in(0,1)$, we can decompose $v$ as $v=v_{0}+\bar{v}$, where

$$
\int_{S^{N-1}} \bar{v} d v o l_{S^{N-1}}=0 \text {. }
$$

The perturbed geodesic ball $B_{1+v}^{\bar{g}}(p)$ can be parameterized by the map $Y_{p, v}: B_{1} \rightarrow B_{1+v}^{\bar{g}}(p)$ given by

$$
Y_{p, v}(x):=\exp _{p}^{\bar{g}}\left(\left(1+v_{0}+\chi(x) \bar{v}(x /|x|)\right) \sum_{i=1}^{N} x^{i} E_{i}\right)
$$


In the following, we will put $\rho:=1+v$ and denote by $\rho_{i}$ (resp. $\rho_{i j}$ ) the partial derivative of $\rho$ with respect to $x^{i}$ (resp. the partial derivatives with respect to $x^{i}$ and $x^{j}$ ).

The parametrization (2.3) induces a metric $\hat{g}$ on $\mathbb{R}^{N}$. Our next task is to derive the Taylor expansion of the metric $\hat{g}$. To this end, we will need to fix some notations.

Notations: Any expression of the form $L_{p}^{i}(v)$ denotes a linear combination of the function $v$ together with its partial derivatives with respect to $x^{i}$ up to order $i=0,1,2$. The coefficient of $L_{p}^{i}$ might depend on $\varepsilon$ and $p$ but, for all $k \in \mathbb{N}$, there exists a constant $c>0$ independent of $\varepsilon$ and $p$ such that

$$
\left\|L_{p}^{i}(v)\right\|_{C^{k, \alpha}\left(S^{N-1}\right)} \leq c\|v\|_{C^{k+i, \alpha}\left(S^{N-1}\right)} .
$$

Similarly, any expression of the form $Q_{p}^{i}(v)$ denotes a nonlinear operator in the function $v$ together with its derivatives with respect to $x^{i}$ up to order $i$. The coefficient of the Taylor expansion of $Q_{p}^{i}(v)$ in power of $v$ and its partial derivatives might depend on $\varepsilon$ and $p$ and, given $k \in \mathbb{N}$, there exists a constant $c>0$ such that $Q_{p}^{i}(0)=0$ and

$$
\begin{gathered}
\left\|Q_{p}^{i}\left(v_{1}\right)-Q_{p}^{i}\left(v_{2}\right)\right\|_{C^{k, \alpha}\left(S^{N-1}\right)} \leq c\left(\left\|v_{1}\right\|_{C^{k+i, \alpha}\left(S^{N-1}\right)}+\left\|v_{1}\right\|_{C^{k+i, \alpha}\left(S^{N-1}\right)}\right) \times \\
\left\|v_{1}-v_{2}\right\|_{C^{k+i, \alpha}\left(S^{N-1}\right)}
\end{gathered}
$$

provided $\left\|v_{1}\right\|_{C^{1, \alpha}\left(S^{N-1}\right)}+\left\|v_{2}\right\|_{C^{1, \alpha}\left(S^{N-1}\right)} \leq 1$. Terms of the form $O_{p}\left(\varepsilon^{l}\right)$ are smooth functions on $S^{N-1}$ that might depend on $p$ but which are bounded by a constant (independent of $p$ ) times $\varepsilon^{l}$ in the $C^{k}$ topology, for all $k \in \mathbb{N}$. Finally the function $P_{\varepsilon}^{i}(v)$ stands for

$$
P_{\varepsilon}^{i}(v)=\varepsilon^{2} L^{i}(v)+Q^{i}(v)+O_{p}\left(\varepsilon^{4}\right) .
$$

We recall that the map $Y_{p, v}$ parameterizes $B_{1+v}^{\bar{g}}(p)$ and we denote by $\hat{g}$ the pull-back metric on $B_{1}$ via $Y_{p, v}$. At the point $q=Y_{p, v}(x)$, we define

$$
\hat{g}_{i j}(x):=\bar{g}\left(\frac{\partial Y_{p, v}}{\partial x^{i}}(x), \frac{\partial Y_{p, v}}{\partial x^{j}}(x)\right) .
$$

Lemma 2.2. For all $x \in B_{1}$, we have the following expansions

$\hat{g}_{i j}(x)=\rho^{2}\left(\delta_{i j}+\rho_{i} x^{j}+\rho_{j} x^{i}+\frac{\varepsilon^{2}}{3} g\left(R_{p}\left(E_{i}, X\right) E_{j}, X\right)+\frac{\varepsilon^{3}}{6} g\left(\nabla_{X} R_{p}\left(E_{i}, X\right) E_{j}, X\right)+P_{\varepsilon}^{1}(v)\right)$ 
and

$$
\begin{aligned}
\Delta_{\hat{g}}= & \rho^{-2} \Delta-2 \sum_{i, j=1}^{N} x^{i} \rho_{j} \partial_{i j}^{2}-2 \sum_{j=1}^{N} \rho_{j} \partial_{j}-\Delta \rho \sum_{j=1}^{N} x^{j} \partial_{j}-\frac{\varepsilon^{2}}{3} \sum_{i, j=1}^{N} g\left(R_{p}\left(E_{i}, X\right) E_{j}, X\right) \partial_{i j}^{2} \\
& +\frac{2 \varepsilon^{2}}{3} \sum_{i, j=1}^{N} g\left(R_{p}\left(E_{i}, E_{j}\right) E_{i}, X\right) \partial_{j}+\frac{\varepsilon^{3}}{3} \sum_{i, j=1}^{N} g\left(\nabla_{X} R_{p}\left(E_{i}, X\right) E_{i}, E_{j}\right) \partial_{j} \\
& -\frac{\varepsilon^{3}}{6} \sum_{i, j=1}^{N} g\left(\nabla_{E_{i}} R_{p}\left(E_{i}, X\right) E_{j}, X\right) \partial_{j}+\frac{\varepsilon^{3}}{12} \sum_{i, j=1}^{N} g\left(\nabla_{E_{j}} R_{p}\left(E_{i}, X\right) E_{i}, X\right) \partial_{j} \\
& -\frac{\varepsilon^{3}}{6} \sum_{i, j=1}^{N} g\left(\nabla_{X} R_{p}\left(E_{i}, X\right) E_{j}, X\right) \partial_{i j}^{2}+\sum_{i, j=1}^{N} \Delta_{\varepsilon, \bar{v}}^{i j},
\end{aligned}
$$

where

$$
\Delta_{\varepsilon, \bar{v}}^{i j}=P_{\varepsilon}^{2}(v) \partial_{i j}+P_{\varepsilon}^{2}(v) \partial_{j}
$$

Proof. We have

$$
\frac{\partial Y_{p, v}}{\partial x^{i}}(x)=\rho_{i} \sum_{k=1}^{N} x^{k} X_{k}+\rho X_{i}=\rho_{i} \Upsilon+\rho X_{i} \quad \forall i=1, \ldots, N
$$

where

$$
\Upsilon=\sum_{k=1}^{N} x^{k} X_{k}
$$

We find using the expansion (2.2) that

$$
\bar{g}(\Upsilon, \Upsilon) \equiv|x|^{2} \quad \text { and } \quad \bar{g}\left(\Upsilon, X_{i}\right) \equiv x^{i}, \quad i=1, \ldots, N .
$$

These equalities then yield

$$
\hat{g}_{i j}=\rho^{2}\left(\delta_{i j}+\rho_{i} x^{j}+\rho_{j} x^{i}+\frac{\varepsilon^{2}}{3} g\left(R_{p}\left(E_{i}, X, E_{j}, X\right)+\frac{\varepsilon^{3}}{6} g\left(\nabla_{X} R_{p}\left(E_{i}, X, E_{j}, X\right)+P_{\varepsilon}^{1}(v)\right) .\right.\right.
$$

The first expansion in the lemma then follows.

The expansion of Laplace-Beltrami operator of the metric $\hat{g}$ is obtained using the formula

$$
\Delta_{\hat{g}}=\hat{g}^{i j} \partial_{i j}^{2}+\left(\partial_{i} \hat{g}^{i j}\right) \partial_{j}+\frac{1}{2} \hat{g}^{i j}\left(\partial_{i} \log |\hat{g}|\right) \partial_{j}=(1)+(2)+(3) .
$$

We start with the last term. Thanks to (2.5), it is not difficult to see that

$\hat{g}^{i j}=\rho^{-2}\left(\delta_{i j}-\rho_{i} x^{j}-\rho_{j} x^{i}-\frac{\varepsilon^{2}}{3} g\left(R_{p}\left(E_{i}, X, E_{j}, X\right)-\frac{\varepsilon^{3}}{6} g\left(\nabla_{X} R_{p}\left(E_{i}, X, E_{j}, X\right)+P_{\varepsilon}^{1}(v)\right)\right.\right.$.

We also have

$$
\log |\hat{g}|=2 N \log \rho+2 \sum_{s=1}^{N} x^{s} \rho_{s}-\frac{\varepsilon^{2}}{3} \operatorname{Ric}_{p}(X, X)+\frac{\varepsilon^{3}}{6} \sum_{s=1}^{N} g\left(\nabla_{X} R_{p}\left(E_{s}, X\right) E_{s}, X\right)+P_{\varepsilon}^{1}(v)
$$


and by a computation, we get

$$
\begin{aligned}
\partial_{i}(\log |\hat{g}|) & =2(N+1) \rho_{i}+2 \sum_{s=1}^{N} x^{s} \rho_{i s}+\frac{2 \varepsilon^{2}}{3} \sum_{k=1}^{N} g\left(R_{p}\left(E_{k}, E_{i}\right) E_{k}, X\right) \\
& +\frac{\varepsilon^{3}}{3} \sum_{s=1}^{N} g\left(\nabla_{X} R_{p}\left(E_{s}, X\right) E_{s}, E_{i}\right)+\frac{\varepsilon^{3}}{6} \sum_{s=1}^{N} g\left(\nabla_{E_{i}} R_{p}\left(E_{s}, X\right) E_{s}, X\right)+P_{\varepsilon}^{2}(v) .
\end{aligned}
$$

This together with (2.7) give

$$
\begin{aligned}
(3) & =(N+1) \sum_{j=1}^{N} \rho_{j} \partial_{j}+\sum_{i j=1}^{N} x^{i} \rho_{i j} \partial_{j}+\frac{\varepsilon^{2}}{3} \sum_{s, j=1}^{N} g\left(R_{p}\left(E_{s}, E_{j}\right) E_{s}, X\right) \partial_{j} \\
& +\frac{\varepsilon^{3}}{6} \sum_{s, j=1}^{N} g\left(\nabla_{X} R_{p}\left(E_{s}, X\right) E_{s}, E_{j}\right) \partial_{j}+\frac{\varepsilon}{12} \sum_{s, j=1}^{N} g\left(\nabla_{E_{j}} R_{p}\left(E_{s}, X\right) E_{s}, X\right) \partial_{j}+\sum_{j=1}^{N} P_{\varepsilon}^{2}(v) \partial_{j} .
\end{aligned}
$$

We compute the partial derivative of $\hat{g}^{i j}$ with respect to $x_{i}$ and get

$$
\begin{aligned}
(2) & =-(N+3) \sum_{j=1}^{N} \rho_{j} \partial_{j}-\Delta \rho \sum_{j=1}^{N} x^{j} \partial_{j}-\sum_{i, j=1}^{N} x^{i} \rho_{i j} \partial_{j}-\frac{\varepsilon^{2}}{3} \sum_{i, j=1}^{N} g\left(R_{p}\left(E_{i}, X\right) E_{j}, E_{i}\right) \partial_{j} \\
& -\frac{\varepsilon^{3}}{6} \sum_{i, j=1}^{N}\left(g\left(\nabla_{E_{i}} R_{p}\left(E_{i}, X\right) E_{j}, X\right)+g\left(\nabla_{X} R_{p}\left(E_{i}, X\right) E_{j}, E_{i}\right)\right) \partial_{j}+P_{\varepsilon}^{2}(v) \sum_{j=1}^{N} \partial_{j} .
\end{aligned}
$$

Therefore

$$
\begin{aligned}
(2)+(3) & =-2 \sum_{j=1}^{N} \rho_{j} \partial_{j}-\Delta \rho \sum_{j=1}^{N} x^{j} \partial_{j}+\frac{2 \varepsilon^{2}}{3} \sum_{i, j=1}^{N} g\left(R_{p}\left(E_{i}, E_{j}\right) E_{i}, X\right) \partial_{j} \\
& +\frac{\varepsilon^{3}}{3} \sum_{i, j=1}^{N}\left(g\left(\nabla_{X} R_{p}\left(E_{i}, X\right) E_{i}, E_{j}\right) \partial_{j}-\frac{\varepsilon^{3}}{6} \sum_{i, j=1}^{N} g\left(\nabla_{E_{i}} R_{p}\left(E_{i}, X\right) E_{j}, X\right) \partial_{j}\right. \\
& +\frac{\varepsilon^{3}}{12} \sum_{i, j=1}^{N} g\left(\nabla_{E_{j}} R_{p}\left(E_{i}, X\right) E_{i}, X\right) \partial_{j}+P_{\varepsilon}^{2}(\bar{v}) \sum_{j=1}^{N} \partial_{j} .
\end{aligned}
$$

Since

$$
\begin{aligned}
(1) & =\rho^{-2} \Delta-2 \sum_{i, j=1}^{N} x^{i} \rho_{j} \partial_{i j}^{2}-\frac{\varepsilon^{2}}{3} \sum_{i, j=1}^{N} g\left(R_{p}\left(E_{i}, X\right) E_{j}, X\right) \partial_{i j}^{2} \\
& -\frac{\varepsilon^{2}}{6} \sum_{i, j=1}^{N}\left(g\left(\nabla_{X} R_{p}\left(E_{i}, X\right) E_{j}, X\right) \partial_{i j}^{2}+P_{\varepsilon}^{2}(\bar{v}) \sum_{i, j=1}^{N} \partial_{i j}^{2},\right.
\end{aligned}
$$


we conclude that

$$
\begin{aligned}
\Delta_{\hat{g}} & =\rho^{-2} \Delta-2 \sum_{i, j=1}^{N} x^{i} \rho_{j} \partial_{i j}^{2}-2 \sum_{j=1}^{N} \rho_{j} \partial_{j}-\Delta \rho \sum_{j=1}^{N} x^{j} \partial_{j}-\frac{\varepsilon^{2}}{3} \sum_{i, j=1}^{N} g\left(R_{p}\left(E_{i}, X\right) E_{j}, X\right) \partial_{i j}^{2} \\
& +\frac{2 \varepsilon^{2}}{3} \sum_{i, j=1}^{N} g\left(R_{p}\left(E_{i}, E_{j}\right) E_{i}, X\right) \partial_{j}+\frac{\varepsilon^{3}}{3} \sum_{i, j=1}^{N} g\left(\nabla_{X} R_{p}\left(E_{i}, X\right) E_{i}, E_{j}\right) \partial_{j} \\
& -\frac{\varepsilon^{3}}{6} \sum_{i, j=1}^{N} g\left(\nabla_{E_{i}} R_{p}\left(E_{i}, X\right) E_{j}, X\right) \partial_{j}+\frac{\varepsilon^{3}}{12} \sum_{i, j=1}^{N} g\left(\nabla_{E_{j}} R_{p}\left(E_{i}, X\right) E_{i}, X\right) \partial_{j} \\
& -\frac{\varepsilon^{3}}{6} \sum_{i, j=1}^{N} g\left(\nabla_{X} R_{p}\left(E_{i}, X\right) E_{j}, X\right) \partial_{i j}^{2}+\sum_{i, j=1}^{N} \Delta_{\varepsilon, \bar{v}}^{i j}
\end{aligned}
$$

as desired.

\section{Construction of Solutions to OVer-Determined Problem}

As explained in the previous section, our aim is to find a point $p$ and a (small) function $v: S^{N-1} \longrightarrow(0, \infty)$ such that the over-determined problem

$$
\begin{cases}-\Delta_{\bar{g}} \bar{u}=1 & \text { in } B_{1+v}^{\bar{g}}(p) \\ \bar{u}=0 & \text { on } \partial B_{1+v}^{\bar{g}}(p) \\ \bar{g}\left(\nabla_{\bar{g}} \bar{u}, \bar{\nu}\right)=\bar{C}_{0} & \text { on } \partial B_{1+v}^{\bar{g}}(p),\end{cases}
$$

has a solution provided $\varepsilon$ is small. In $\mathbb{R}^{N}$ a solution is given by

$$
\phi_{0}(x):=\frac{1-|x|^{2}}{2 N}
$$

which clearly satisfies

$$
\begin{cases}-\Delta \phi_{0}=1 & \text { in } B_{1} \\ \phi_{0}=0 & \text { on } \partial B_{1} \\ \frac{\partial \phi_{0}}{\partial \nu}=-\frac{1}{N} & \text { on } \partial B_{1} .\end{cases}
$$

The next result provides a first approximate solution to (3.1) by solving only the Dirichlet problem in (3.1).

Proposition 3.1. There exists $\varepsilon_{0}>0$ such that for all $\varepsilon \in\left(0, \varepsilon_{0}\right)$, for $p \in \mathcal{M}$ and for all $\left(v_{0}, \bar{v}\right) \in \mathbb{R} \times C^{2, \alpha}\left(S^{N-1}\right)$ satisfying

$$
\left|v_{0}\right| \leq \varepsilon_{0},\|\bar{v}\|_{C^{2, \alpha}\left(S^{n-1}\right)} \leq \varepsilon_{0} \quad \text { and } \quad \int_{S^{N-1}} \bar{v} d v o l_{S^{N-1}}=0
$$


there exists a unique positive function $\bar{\phi}=\bar{\phi}\left(p, \varepsilon, v_{0}, \bar{v}\right) \in C^{2, \alpha}\left(\overline{B_{1+v}^{\bar{g}}(p)}\right)$ such that

$$
\begin{cases}-\Delta_{\bar{g}} \bar{\phi}=1 & \text { in } B_{1+v}^{\bar{g}}(p) \\ \bar{\phi}=0 & \text { on } \partial B_{1+v}^{\bar{g}}(p) .\end{cases}
$$

The function $\bar{\phi}$ depends smoothly on $v_{0}, \bar{v}, \varepsilon$. In addition $\bar{\phi}=\phi_{0}$ when $\varepsilon=0, v_{0}=0$ and $\bar{v} \equiv 0$.

Proof. By change of variables, (3.3) is equivalent to

$$
\begin{cases}-\Delta_{\hat{g}} \hat{\phi}=1 & \text { in } B_{1} \\ \hat{\phi}=0 & \text { on } \partial B_{1},\end{cases}
$$

where $\hat{g}$ is the induced metric defined in Lemma 2.2 .

Observe that, when $\varepsilon=0, v_{0}=0$ and $\bar{v} \equiv 0, \hat{g}$ is the Euclidean metric $g_{0}$ and the solution of (3.4) is given by $\hat{\phi}=\phi_{0}$. In fact the solution of (3.4) is the pull-back of the solution of (3.3) via the parametrization $Y_{p, v}$. We mean by this, $\hat{\phi}=Y_{p, v}^{*} \bar{\phi}$.

Define the Banach spaces

$$
C_{\text {Dir }}^{2, \alpha}\left(\overline{B_{1}}\right):=\left\{u \in C^{2, \alpha}\left(\overline{B_{1}}\right) \quad: \quad u=0 \quad \text { on } \partial B_{1}\right\}
$$

and

$$
C_{m}^{2, \alpha}\left(S^{N-1}\right):=\left\{v \in C_{m}^{2, \alpha}\left(S^{N-1}\right), \int_{S^{N-1}} v \operatorname{dvol}_{S^{N-1}}=0\right\} .
$$

Now consider the map

$$
\begin{array}{r}
\mathcal{N}:[0, \infty) \times \mathbb{R} \times C_{m}^{2, \alpha}\left(S^{N-1}\right) \times C_{D i r}^{2, \alpha}\left(B_{1}\right) \longrightarrow C^{0, \alpha}\left(\overline{B_{1}}\right) \\
\left(\varepsilon, v_{0}, \bar{v}, \phi\right) \longmapsto \Delta_{\hat{g}} \phi+1,
\end{array}
$$

It is clear that

$$
\mathcal{N}\left(0,0,0, \phi_{0}\right)=0
$$

and $\mathcal{N}$ is a smooth map in a neighborhood of $\left(0,0,0, \phi_{0}\right)$ in $[0, \infty) \times \mathbb{R} \times C_{m}^{2, \alpha}\left(S^{N-1}\right) \times$ $C_{D i r}^{2, \alpha}\left(\overline{B_{1}}\right)$. Now since $\partial_{\phi} \mathcal{N}\left(0,0,0, \phi_{0}\right)=\Delta: C_{D i r}^{2, \alpha}\left(\overline{B_{1}}\right) \rightarrow C^{0, \alpha}\left(\overline{B_{1}}\right)$ is invertible, the implicit function theorem gives the desired result.

Our next task is to prove that it is possible to find $\left(p, \varepsilon, v_{0}, \bar{v}\right)$ such that

$$
\bar{g}\left(\nabla_{\bar{g}} \bar{\phi}, \bar{\nu}\right)=-\frac{1}{N} \quad \text { on } \quad \partial B_{1+v}^{\bar{g}}(p)
$$

We compute the Taylor of $\bar{g}\left(\nabla_{\bar{g}} \bar{\phi}, \bar{\nu}\right)$. To this end, we need an accurate approximation $\bar{\phi}$ which is given by Proposition 3.1. We define $\hat{\phi}=\hat{\phi}(\varepsilon, p, v): B_{1} \rightarrow \mathbb{R}$ by

$$
\hat{\phi}(x):=\bar{\phi}\left(Y_{p, v}(x)\right)=\phi_{0}(\rho x)+\Psi_{\varepsilon, v}(x) \quad \forall x \in B_{1},
$$


where we recall that $\rho=1+\left(v_{0}+\chi v\right)$. By $(3.4)$, the function $\Psi_{\varepsilon, v}$ satisfies

$$
\left\{\begin{array}{l}
-\Delta_{\hat{g}} \Psi_{\varepsilon, v}=1+\Delta_{\hat{g}} \phi_{0}(\rho x) \quad \text { in } B_{1} \\
\Psi_{\varepsilon, v}=-\phi_{0}(\rho x) \quad \text { on } \partial B_{1} .
\end{array}\right.
$$

The expansion of $\phi_{0}(\rho x)$ is given by

$$
\phi_{0}(\rho x)=\phi_{0}(x)-\frac{1}{N}|x|^{2}(\rho-1)+Q_{p}^{0}(v)
$$

and we have

Lemma 3.2. The function $\Psi_{\varepsilon, v}$ defined in (3.6) satisfies

$$
\left\{\begin{array}{cc}
-\Delta \Psi_{\varepsilon, v}=\frac{\varepsilon^{2}}{3 N} & \operatorname{Ric}_{p}(X, X)-\frac{\varepsilon^{3}}{4 N} \sum_{i=1}^{N} g\left(\nabla_{X} R_{p}\left(E_{i}, X\right) E_{i}, X\right) \\
+\frac{\varepsilon^{3}}{6 N} \sum_{i=1}^{N} g\left(\nabla_{E_{i}} R_{p}\left(E_{i}, X\right) X, X\right)+P_{\varepsilon}^{2}(v) & \text { in } B_{1} \\
\Psi_{\varepsilon, v}=\frac{1}{N} v+Q_{p}^{0}(v) & \text { on } \partial B_{1} .
\end{array}\right.
$$

Proof. By straightforward computations using (3.8) and the expansion of $\Delta_{\hat{g}}$ in Lemma 2.2 , we get for all $x \in B_{1}$,

$$
\begin{aligned}
\Delta_{\hat{g}} \phi_{0}(x) & =-\rho^{-2}+\frac{4}{N}\langle\nabla \rho, x\rangle+\frac{1}{N}|x|^{2} \Delta \rho+\frac{\varepsilon^{2}}{3 N} \operatorname{Ric}_{p}(X, X) \\
& -\frac{\varepsilon^{3}}{4 N} \sum_{i=1}^{N} g\left(\nabla_{X} R_{p}\left(E_{i}, X\right) E_{i}, X\right)+\frac{\varepsilon^{3}}{6 N} \sum_{i=1}^{N} g\left(\nabla_{E_{i}} R_{p}\left(E_{i}, X\right) X, X\right)+P_{\varepsilon}^{2}(v) .
\end{aligned}
$$

Similarly, using Lemma 2.2, we have

$$
-\Delta_{\hat{g}}\left(\frac{1}{N}|x|^{2}(\rho-1)\right)=-\Delta\left(\frac{1}{N}|x|^{2}(\rho-1)\right)+P_{\varepsilon}^{2}(v)=-\frac{1}{N}|x|^{2} \Delta \rho-\frac{4}{N}\langle\nabla \rho, x\rangle-2 v+P_{\varepsilon}^{2}(v) .
$$

From the two previous inequalities and (3.7), we deduce the first equality of (3.9). Finally using (3.6), (3.8) and the fact that $\hat{\phi}$ and $\phi_{0}$ are equal to 0 on $\partial B_{1}$, we obtain

$$
\Psi_{\varepsilon, v}=\frac{1}{N} v+Q_{p}^{0}(v) \quad \text { on } \partial B_{1} .
$$

Lemma 3.3. At a point $x \in \partial B_{1}$, we have the expansion

$$
\hat{g}\left(\nabla_{\hat{g}} \hat{\phi}, \hat{\nu}\right)_{\left.\right|_{\partial B_{1}}}=-\frac{1}{N}+\frac{1}{N}\left[\left(\partial_{\nu} \psi_{v}\right)_{\left.\right|_{\partial B_{1}}}-v\right]+\left(\partial_{\nu} \psi_{\varepsilon}\right)_{\left.\right|_{\partial B_{1}}}+\left(\partial_{\nu} \Gamma_{\varepsilon, v}\right)_{\left.\right|_{\partial B_{1}}}+P_{\varepsilon}^{1}(v),
$$

where the functions $\psi_{v}, \psi_{\varepsilon}$ and $\Gamma_{\varepsilon, v}$ are respectively (unique) solution to

$$
\begin{cases}\Delta \psi_{v}=0 & \text { in } B_{1} \\ \psi_{v}=v & \text { on } \partial B_{1},\end{cases}
$$




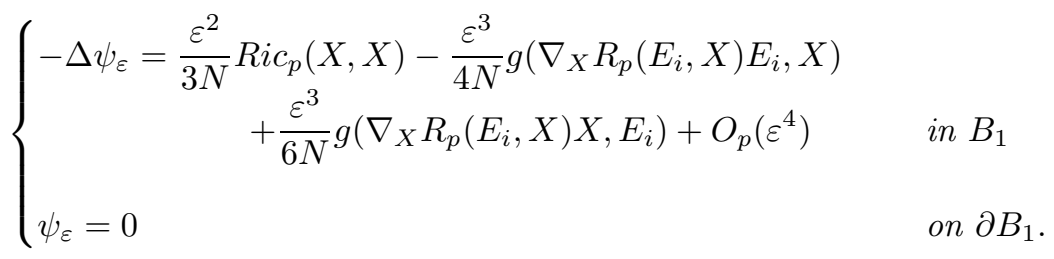

and

$$
\left\{\begin{array}{l}
\Delta \Gamma_{\varepsilon, v}=P_{\varepsilon}^{2}(v) \quad \text { in } B_{1} \\
\Gamma_{\varepsilon, v}=Q^{0}(v) \quad \text { on } \partial B_{1},
\end{array}\right.
$$

Proof. Since $\hat{\phi}=0$ on $B_{1}$, the unit outward vector $\hat{\nu}$ about $\partial B_{1}$ is given by

$$
\hat{\nu}=-\frac{\nabla_{\hat{g}} \hat{\phi}}{\left|\nabla_{\hat{g}} \hat{\phi}\right|_{\hat{g}}}
$$

and thus

$$
\hat{g}\left(\nabla_{\hat{g}} \hat{\phi}, \hat{\nu}\right)=-\left|\nabla_{\hat{g}} \hat{\phi}\right|_{\hat{g}}
$$

From the expansion of $\hat{g}$ in Lemma 2.2, we have

$$
\begin{aligned}
\left|\nabla_{\hat{g}} \hat{\phi}\right|_{\hat{g}}^{2} & =\hat{g}\left(\nabla_{\hat{g}} \hat{\phi}, \nabla_{\hat{g}} \bar{\phi}\right)=\sum_{i l=1}^{N} \hat{g}^{i l}(x) \frac{\partial \hat{\phi}}{\partial x^{i}} \frac{\partial \hat{\phi}}{\partial x^{l}}+P_{\varepsilon}^{1}(v)=\rho^{-2} \sum_{i=1}^{N}\left(\frac{\partial \hat{\phi}}{\partial x^{i}}\right)^{2}+P_{\varepsilon}^{1}(v) \\
& =\rho^{-2}|\nabla \hat{\phi}|^{2}+P_{\varepsilon}^{1}(v) .
\end{aligned}
$$

We also have

$$
\hat{\phi}=\phi_{0}(\rho x)+\Psi_{\varepsilon, v} \text { and } \quad \partial_{j} \phi_{0}(\rho x)=-\frac{1}{N}\left(|x|^{2} \rho_{j}+x^{j} \rho^{2}\right), j=1, \ldots, N .
$$

This implies

$$
\hat{g}\left(\nabla_{\hat{g}} \hat{\phi}, \hat{\nu}\right)_{\left.\right|_{\partial B_{1}}}=-\rho^{-1}|\nabla \hat{\phi}|+P_{\varepsilon}^{1}(v)=-\frac{1}{N}-\frac{1}{N} v+\left(\partial_{\nu} \Psi_{\varepsilon, v}\right)_{\left.\right|_{\partial B_{1}}}+P_{\varepsilon}^{1}(v) .
$$

Recalling Lemma 3.2 , we can decompose $\Psi_{\varepsilon, v}$ as

$$
\Psi_{\varepsilon, v}=\frac{1}{N} \psi_{v}+\psi_{\varepsilon}+\Gamma_{\varepsilon, v},
$$

where the functions $\psi_{\bar{v}}, \psi_{\varepsilon}$ and $\Gamma_{\varepsilon, v}$ are respectively (unique) solution of

$$
\begin{gathered}
\begin{cases}\Delta \psi_{v}=0 & \text { in } B_{1} \\
\psi_{v}=v & \text { on } \partial B_{1},\end{cases} \\
\left\{\begin{aligned}
-\Delta \psi_{\varepsilon}=\frac{\varepsilon^{2}}{3 N} R i c_{p}(X, X)-\frac{\varepsilon^{3}}{4 N} g\left(\nabla_{X} R_{p}\left(E_{i}, X\right) E_{i}, X\right) & \text { in } B_{1} \\
+\frac{\varepsilon^{3}}{6 N} g\left(\nabla_{X} R_{p}\left(E_{i}, X\right) X, E_{i}\right)+O_{p}\left(\varepsilon^{4}\right) & \text { on } \partial B_{1}
\end{aligned}\right.
\end{gathered}
$$


and

$$
\begin{cases}\Delta \Gamma_{\varepsilon, v}=P_{\varepsilon}^{2}(v) & \text { in } B_{1} \\ \Gamma_{\varepsilon, v}=Q^{0}(v) & \text { on } \partial B_{1} .\end{cases}
$$

We define

$$
G\left(p, \varepsilon, v_{0}, \bar{v}\right):=\frac{1}{N}\left[\left(\partial_{\nu} \psi_{v}\right)_{\left.\right|_{\partial B_{1}}}-v\right]+\left(\partial_{\nu} \psi_{\varepsilon}\right)_{\left.\right|_{\partial B_{1}}}+\left(\partial_{\nu} \Gamma_{\varepsilon, v}\right)_{\left.\right|_{\partial B_{1}}}+P_{\varepsilon}^{1}(v),
$$

so that,

$$
\left.\bar{g}\left(\nabla_{\bar{g}} \bar{\phi}, \bar{\nu}\right)\right|_{\partial B_{1+v}^{\bar{g}}}=\hat{g}\left(\nabla_{\hat{g}} \hat{\phi}, \hat{\nu}\right)_{\left.\right|_{\partial B_{1}}}=-\frac{1}{N}+G\left(p, \varepsilon, v_{0}, \bar{v}\right)
$$

and thus our objective (3.5) then becomes to find $\left(p, \varepsilon, v_{0}, \bar{v}\right)$ such that $G\left(p, \varepsilon, v_{0}, \bar{v}\right)=0$.

To solve this, we will use variational perturbative methods keeping in mind that the sets we are looking for are stationary sets for some energy functional. The main strategy consists first in using a local inversion argument to reduce the problem to finite dimensional critical point problem. This is due to the fact that the problem under study is invariant by translations on $\mathbb{R}^{N}$ and so the energy has a "kernel" at least of dimension $N$. This phenomenon brings some difficulties to invert the map $\bar{v} \mapsto \frac{\partial}{\partial \bar{v}} G(p, 0,0,0)$ as it might have zero eigenvalues. However, as we shall see, $\operatorname{Ker} \frac{\partial}{\partial \bar{v}} G(p, 0,0,0)=\left\{x^{i}, \quad i=1, \ldots, N\right\}$. Therefore we will solve (3.5) modulo this set by local inversion theorems. This is the aim of the next section.

3.1. Local inversion argument. Let us consider the Dirichlet-to-Neumann operator

$$
v \mapsto\left(\partial_{\nu} \psi_{v}\right)_{\partial_{B_{1}}},
$$

where

$$
\left\{\begin{array}{l}
\Delta \psi_{v}=0 \quad \text { in } B_{1} \\
\psi_{v}=v \quad \text { on } \partial B_{1} .
\end{array}\right.
$$

It is well known, see for instance [38], that this map has a discrete spectrum in $L^{2}\left(S^{N-1}\right)$ given by

$$
\lambda_{k}=k, \quad k \in \mathbb{N}
$$

which corresponds to the Steklov eigenvalue problem. The eigenvectors corresponding to the eigenvalue $\lambda_{k}$ are given by the spherical harmonics $Y_{k}$ which satisfy $-\Delta_{S^{N-1}} Y_{k}=k(k+$ $N-2) Y_{k}$ on $S^{N-1}$. Therefore the eigenspaces corresponding to $\lambda_{0}=0$ and $\lambda_{1}=1$ are

$$
\Lambda_{0}:=\operatorname{span}\{1\} \quad \text { and } \quad \Lambda_{1}:=\operatorname{span}\left\{x^{1}, \cdots, x^{N}\right\}
$$

respectively. We denote by $\Pi_{0}$ and $\Pi_{1}$ the $L^{2}$ projections onto these spaces respectively and we define

$$
\Pi:=\mathrm{Id}-\Pi_{1}-\Pi_{0} \quad \text { and } \quad \Pi_{1}^{\perp}:=\Pi_{0}+\Pi .
$$

Combining these with elliptic regularity theory, we have the following 
Proposition 3.4. We define the operator $\mathbb{L}(v):=\left(\partial_{\nu} \psi_{v}\right)_{\left.\right|_{\partial B_{1}}}-v$. Then

$$
\mathbb{L}: C^{2, \alpha}\left(S^{N-1}\right) \longrightarrow C^{1, \alpha}\left(S^{N-1}\right)
$$

is a self adjoint, first order elliptic operator. In addition

$$
K e r \mathbb{L}=\left\{x^{i}, \quad i=1, \ldots, N\right\} .
$$

Moreover there exists $c>0$ such that

$$
\|w\|_{C^{2, \alpha}\left(S^{N-1}\right)} \leq c\|\mathbb{L}(w)\|_{C^{1, \alpha}\left(S^{N-1}\right)}
$$

for every $w \in \Pi_{1}^{\perp} C^{2, \alpha}\left(S^{N-1}\right)$.

We are now able to prove that, for $\varepsilon$ small enough, it is possible to solve equation

$$
G\left(p, \varepsilon, v_{0}, \bar{v}\right)=0
$$

modulo the kernel of $\mathbb{L}=\frac{\partial}{\partial v} G(p, 0,0,0)$. Indeed we have

Proposition 3.5. There exists $\varepsilon_{0}>0$ such that, for all $\varepsilon \in\left(0, \varepsilon_{0}\right)$ and for all $p \in \mathcal{M}$ there exists a unique $v^{\varepsilon, p} \in C^{2, \alpha}\left(S^{N-1}\right)$ with

$$
\left\|v^{\varepsilon, p}\right\|_{C^{2, \alpha}\left(S^{N-1}\right)}<\varepsilon_{0}
$$

such that $\hat{\phi}^{\varepsilon, p}=\hat{\phi}\left(\varepsilon, p, v_{0}^{\varepsilon, p}, \bar{v}^{\varepsilon, p}\right)$ satisfies

$$
\begin{cases}-\Delta_{\hat{g}} \hat{\phi}^{\varepsilon, p}=1 & \text { in } B_{1} \\ \hat{\phi}^{\varepsilon, p}=0 & \text { on } \partial B_{1} \\ \hat{g}\left(\nabla_{\hat{g}} \hat{\phi}^{\varepsilon, p}, \hat{\nu}\right)=-\frac{1}{N}-\left\langle a^{\varepsilon, p}, x\right\rangle & \text { on } \partial B_{1}\end{cases}
$$

where $v_{0}^{\varepsilon, p}=\Pi_{0} v^{\varepsilon, p},\left\langle a^{\varepsilon, p}, x\right\rangle=\Pi_{1} v^{\varepsilon, p}$ and $\bar{v}^{\varepsilon, p}=\Pi v^{\varepsilon, p}$.

In addition the mapping $(\varepsilon, p, x) \mapsto v^{\varepsilon, p}(x)$ is smooth and satisfies

$$
\left\|\nabla_{g}^{k} v^{\varepsilon, p}\right\|_{C^{2, \alpha}\left(T \mathcal{M} \times S^{N-1}\right)} \leq c_{k} \varepsilon^{2},
$$

for all $k \in \mathbb{N}$.

Proof. We consider the map

$$
\mathcal{G}: \mathcal{M} \times\left[0, \infty\left[\times C^{2, \alpha}\left(S^{N-1}\right) \rightarrow C^{1, \alpha}\left(S^{n-1}\right)\right.\right.
$$

given by

$$
\mathcal{G}(p, \varepsilon, v)=G\left(p, \varepsilon, \Pi_{0} v, \Pi v\right)+\Pi_{1} v .
$$

Direct computations show that

$$
\frac{\partial \mathcal{G}}{\partial v}(p, 0,0)[w]=\frac{1}{N} \mathbb{L} \circ \Pi_{1}^{\perp}(w)+\Pi_{1} w .
$$

We define

$$
\mathcal{L}:=\frac{1}{N} \mathbb{L} \circ \Pi_{1}^{\perp}+\Pi_{1}
$$


Thanks to Proposition 3.4, the operator

$$
\mathcal{L}: C^{2, \alpha}\left(S^{N-1}\right) \rightarrow C^{1, \alpha}\left(S^{N-1}\right)
$$

is an isomorphism and for all $w \in C^{2, \alpha}\left(S^{N-1}\right)$

$$
\|w\|_{C^{2, \alpha}\left(S^{N-1}\right)} \leq c\|\mathcal{L}(w)\|_{C^{1, \alpha}\left(S^{N-1}\right)}
$$

Hence the implicit function theorem ensures that there exists $\varepsilon_{0}>0$ such that for all $\varepsilon \in\left(0, \varepsilon_{0}\right)$ and for all $p \in \mathcal{M}$, the existence of a unique $v^{\varepsilon, p} \in C^{2, \alpha}\left(S^{N-1}\right)$ with

$$
\left\|v^{\varepsilon, p}\right\|_{C^{2, \alpha}\left(S^{N-1}\right)}<\varepsilon_{0}
$$

such that

$$
\mathcal{G}\left(p, \varepsilon, v^{\varepsilon, p}\right)=G\left(p, \varepsilon, \Pi_{0} v^{\varepsilon, p}, \Pi v^{\varepsilon, p}\right)+\Pi_{1} v^{\varepsilon, p}=0 .
$$

Recalling (3.15), this is clearly equivalent to

$$
\mathcal{L}\left(v^{\varepsilon, p}\right)+\left(\partial_{\nu} \psi_{\varepsilon}\right)_{\left.\right|_{\partial B_{1}}}+\left(\partial_{\nu} \Gamma_{\varepsilon, v^{\varepsilon, p}}\right)_{\left.\right|_{\partial B_{1}}}+P_{\varepsilon}^{1}\left(v^{\varepsilon, p}\right)=0 .
$$

By elliptic regularity theory

$$
\left\|\Gamma_{\varepsilon, v^{\varepsilon, p}}\right\|_{C^{2, \alpha}\left(S^{N-1}\right)} \leq C \varepsilon^{4}+C \varepsilon^{2}\left\|v^{\varepsilon, p}\right\|_{C^{2, \alpha}\left(S^{N-1}\right)}+C\left\|v^{\varepsilon, p}\right\|_{C^{2, \alpha}\left(S^{N-1}\right)}^{2} .
$$

Decreasing $\varepsilon_{0}$ if necessary, we deduce from (3.21) and (3.22) that

$$
\left\|v^{\varepsilon, p}\right\|_{C^{2, \alpha}\left(S^{N-1}\right)} \leq c \varepsilon^{2} .
$$

The smooth dependence on $\varepsilon, p$ is a consequence of the implicit function theorem. Also (3.20) is a consequence of the fact that $v^{\varepsilon, p}$ solves the differential equation (3.22) (which can be differentiated $k$ times with respect to $p$ ) and the smooth dependence of the metric $\hat{g}$ with respect to $p$ and $\varepsilon$.

3.2. Geometric variational argument. Let $\Omega \subset \mathcal{M}$ be a smooth bounded domain of $\mathcal{M}$. It is very well known that the minimization problem

$$
J(\Omega):=\inf \left\{\int_{\Omega}\left|\nabla_{g} u\right|^{2} \operatorname{dvol}_{g}: \int_{\Omega} u \operatorname{dvol}_{g}=1, u \in H_{0}^{1}(\Omega)\right\} .
$$

has a unique solution $u_{\Omega} \in H_{0}^{1}(\Omega)$ where $J(\Omega)$ is achieved and we have

$$
\begin{cases}-\Delta u_{\Omega}=J(\Omega) & \text { in } \Omega \\ u_{\Omega}=0 & \text { on } \partial \Omega .\end{cases}
$$

We can now consider the functional $\Omega \longmapsto J(\Omega)$, for every bounded and smooth domain $\Omega \subset \mathcal{M}$.

Definition 3.6. We say that $\left\{\Omega_{s}\right\}_{s \in\left[0, s_{0}\right)}$ is a deformation of $\Omega_{0}$, if there exists a vector field $\Xi$ such that $\Omega_{s}=\xi\left(s, \Omega_{0}\right)$, where $\xi(s,$.$) is the flow associated to \Xi$, namely

$$
\xi(0, .)=p \quad \text { and } \quad \frac{d \xi}{d s}(s, p)=\Xi(\xi(s, p)) .
$$

The deformation is volume preserving if $\left|\Omega_{s}\right|_{g}=\left|\Omega_{0}\right|_{g}$ for all $t \in\left[0, s_{0}\right)$. 
Let $\left\{\Omega_{s}\right\}_{s \in\left[0, s_{0}\right)}$ be a deformation of a domain $\Omega_{0}$ generated by the vector field $\Xi$. We denote by $J_{s}=J\left(\Omega_{s}\right)$ Dirichlet's energy define in (3.23), $u_{s}$ the corresponding minimizer on $\Omega_{s}$ and $\nu_{s}$ the outward unit vector field about $\partial \Omega_{s}$. We have the following lemma.

Lemma 3.7. The derivative of $s \longmapsto J_{s}$ at $s=0$ is given by

$$
\left.\frac{d J_{s}}{d s}\right|_{s=0}=-\int_{\partial \Omega_{0}}\left[g\left(\nabla_{g} u_{0}, \nu_{0}\right)\right]^{2} g\left(\Xi, \nu_{0}\right) d \sigma_{g},
$$

where $d \sigma_{g}$ is the volume element on $\partial \Omega_{0}$ for the metric induced by $g$ and $\nu_{0}$ the normal vector field about $\partial \Omega_{0}$. The domain $\Omega_{0}$ is said a stationary set for $J$ if

$$
\left.\frac{d J_{s}}{d s}\right|_{s=0}=0
$$

Proof. We differentiate

$$
-\Delta_{g} u_{s}=J_{s} \quad \text { in } \quad \Omega_{s}
$$

with respect to $s$ and evaluate the result at $s=0$ to obtain

$$
-\Delta_{g} \partial_{s} u_{0}=J_{0}^{\prime} \quad \text { in } \quad \Omega_{0} .
$$

We also know that

$$
\int_{\Omega_{s}} u_{s} \operatorname{dvol}_{g}=1, \quad \text { for all } s \in\left[0, s_{0}\right) .
$$

Differentiating (3.27) with respect to $s$ and evaluating at $s=0$ yields

$$
\int_{\Omega_{s}} \partial_{s} u_{0} \operatorname{dvol}_{g}=0
$$

We multiply (3.26) by $u_{0}$ and (3.25), evaluated at $s=0$, by $\partial_{s} u_{0}$, subtract the two results and integrate over $\Omega_{0}$ to get

$$
\begin{aligned}
J_{0}^{\prime} \int_{\Omega_{0}} u_{0} \operatorname{dvol}_{g}-J_{0} \int_{\Omega_{0}} \partial_{s} u_{0} \operatorname{dvol}_{g} & =\int_{\Omega_{0}}\left(\partial_{s} u_{0} \Delta_{g} u_{0}-u_{0} \Delta_{g} \partial_{s} u_{0}\right) \operatorname{dvol} g \\
& =\int_{\partial \Omega_{0}}\left(\partial_{s} u_{0} g\left(\nabla_{g} u_{0}, \nu_{0}\right)-u_{0} \frac{\partial\left(\partial_{s} u_{0}\right)}{\partial \nu_{0}}\right) d \sigma_{g} \\
& =\int_{\partial \Omega_{0}} \partial_{s} u_{0} g\left(\nabla_{g} u_{0}, \nu_{0}\right) d \sigma_{g}
\end{aligned}
$$

where we have used the fact that $u_{0}=0$ on $\partial \Omega_{0}$ to obtain the last equality. We conclude with (3.27) and (3.28) that

$$
J_{0}^{\prime}=\int_{\partial \Omega_{0}} \partial_{s} u_{0} g\left(\nabla_{g} u_{0}, \nu_{0}\right) d \sigma_{g}
$$

Now, let $\xi$ be the flow generated by $\Xi$, by definition

$$
u_{s}(\xi(s, p))=0 \quad \text { for } p \in \partial \Omega_{s} .
$$

We differentiate (3.30) with respect to $t$ and evaluating at $s=0$ and get

$$
\partial_{s} u_{0}=-g\left(\nabla_{g} u_{0}, \Xi\right) .
$$


But, $u_{0}=0$ on $\partial \Omega_{0}$, and hence only the normal component of $\Xi$ plays a role in this formula. Therefore, we have

$$
\partial_{s} u_{0}=-g\left(\nabla_{g} u_{0}, \nu_{0}\right) g\left(\Xi, \nu_{0}\right) \text { on } \partial \Omega_{0}
$$

and replacing this in (3.29), we finally get that

$$
J_{0}^{\prime}=-\int_{\partial \Omega_{0}}\left[g\left(\nabla_{g} u_{0}, \nu_{0}\right)\right]^{2} g\left(\Xi, \nu_{0}\right) d \sigma_{g} .
$$

The following proposition gives a necessary and sufficient condition for a domain $\Omega$ being a stationary set of $J$.

Proposition 3.8. A domain $\Omega$ is a stationary set for $J$ under volume preserving deformations if and only if their exits a function $u_{\Omega_{0}}$ such that

$$
\begin{cases}-\Delta_{g} u_{\Omega_{0}}=J\left(\Omega_{0}\right) & \text { in } \Omega_{0} \\ u_{\Omega_{0}}=0 & \text { on } \partial \Omega_{0} \\ g\left(\nabla_{g} u_{\Omega_{0}}, \nu_{0}\right)=\lambda & \text { on } \partial \Omega_{0},\end{cases}
$$

for some $\lambda \in \mathbb{R}$.

The proof of this proposition is similar to the one of [Proposition 2.2 in [36]] so we skip it.

We also remark that instead of considering volume preserving deformation, a smooth bounded stationary set $\Omega_{0}$ for the total energy

$$
\Omega \mapsto J(\Omega)+\lambda^{2}|\Omega|_{g}
$$

implies the existence of $u_{\Omega_{0}}$ such that (3.31) holds. This can be seen from Lemma 3.7 and the variation of volume which is given by

$$
\left.\frac{d}{d s}\left|\Omega_{s}\right|_{g}\right|_{s=0}=\int_{\partial \Omega_{0}} g\left(\Xi, \nu_{0}\right) d \sigma_{g}
$$

See for instance [ [22], Theorem 1.11].

3.2.1. The reduced functional. Let us recall what we have obtained so far. Thanks to Propo-

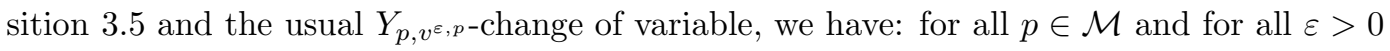
small we have $\bar{\phi}^{\varepsilon, p}=\hat{\phi}^{\varepsilon, p} \circ Y_{p, v^{\varepsilon, p}}^{-1}$ satisfies

$$
\begin{cases}-\Delta_{\bar{g}} \bar{\phi}^{\varepsilon, p}=1 & \text { in } B_{1+v^{\varepsilon, p}}^{\bar{g}}(p) \\ \bar{\phi}^{\varepsilon, p}=0 & \text { on } \partial B_{1+v^{\varepsilon, p}}^{\bar{g}}(p) \\ \bar{g}\left(\nabla_{\bar{g}} \bar{\phi}^{\varepsilon, p}, \bar{\nu}\right)=-\frac{1}{N}-\bar{g}\left(A^{\varepsilon, p}, \mathcal{V}^{\varepsilon, p}\right) & \text { on } \partial B_{1+v^{\varepsilon, p}}^{\bar{g}}(p),\end{cases}
$$


where for all $x \in S^{N-1}$, we define $A^{\varepsilon, p}\left(Y_{p, v^{\varepsilon, p}}(x)\right):=d Y_{p, v^{\varepsilon, p}}(x)\left[a^{\varepsilon, p}\right]$ and similarly $\mathcal{V}^{\varepsilon, p}:=$ $d Y_{p, v^{\varepsilon, p}}(x)[x]$. It follows that the inverse of the torsion rigidity for $\bar{\phi}^{\varepsilon, p}$ is given by

$$
J\left(B_{1+v^{\varepsilon, p}}^{\bar{g}}(p)\right)=\frac{1}{\int_{B_{1+v^{\varepsilon, p}}^{\bar{g}}(p)} \bar{\phi}^{\varepsilon, p} \operatorname{dvol}_{\bar{g}}} .
$$

The domains $\Omega_{\varepsilon}$ we are looking for is a critical point of the the total energy functional :

$$
\Omega \mapsto J(\Omega)+\frac{1}{N^{2}}|\Omega|_{\bar{g}}
$$

This allows to define for $p \in \mathcal{M}$, the reduced functional

$$
\Phi_{\varepsilon}(p):=J\left(B_{1+v^{\varepsilon, p}}^{\bar{g}}(p)\right)+\frac{1}{N^{2}}\left|B_{1+v^{\varepsilon, p}}^{\bar{g}}(p)\right|_{\bar{g}} .
$$

Proposition 3.9. Let $\bar{\phi}^{\varepsilon, p}$ satisfies (3.32). If $p$ is a critical point of $\Phi_{\varepsilon}$ then $A^{\varepsilon, p}=0$, provided $\varepsilon$ is small. In particular

$$
\begin{cases}-\Delta_{\bar{g}} \bar{\phi}^{\varepsilon, p}=1 & \text { in } B_{1+v^{\varepsilon, p}}^{\bar{g}}(p) \\ \bar{\phi}^{\varepsilon, p}=0 & \text { on } \partial B_{1+v^{\varepsilon, p}}^{\bar{g}}(p) \\ \bar{g}\left(\nabla_{\bar{g}} \bar{\phi}^{\varepsilon, p}, \bar{\nu}\right)=-\frac{1}{N} & \text { on } \partial B_{1+v^{\varepsilon, p}}^{\bar{g}}(p) .\end{cases}
$$

Proof. Given $\Xi \in T_{p} \mathcal{M}$, we consider the geodesic curve $p_{s}=\exp _{p}(s \Xi)$. Let $E_{i}^{s}$ be the parallel transport of $E_{i}$ to $p_{s}$ along the curve $[0,1] \ni t \mapsto \exp _{p}^{\bar{g}}\left(t s E_{i}\right)$. Provided $s$ is fixed and small, we can consider the perturbed ball $B_{1+v^{\varepsilon, p_{s}}}^{\bar{g}}\left(p_{s}\right)$ so that (3.32) holds. Recall that

$$
B_{1+v^{\varepsilon, p_{s}}}^{\bar{g}}\left(p_{s}\right)=Y_{v^{\varepsilon, p_{s}, p_{s}}}\left(B_{1}\right) \text {. }
$$

Define the vector field

$$
W_{s}\left(Y_{p, v^{\varepsilon, p}}(x)\right)=\left(1+v^{\varepsilon, p_{s}}(x)\right) \sum_{i=1}^{N} x^{i} E_{i}^{s} \quad \forall x \in B_{1} .
$$

We now define the deformation of $B_{1+v^{\varepsilon, p}}^{\bar{g}}(p)$ by

$$
\xi(s, q)=\exp _{p_{s}}\left(W_{s}(q)\right) \quad \forall q \in Y_{p, v^{\varepsilon, p}}\left(B_{1}\right) .
$$

Next we observe that

$$
\frac{d \xi}{d s}(0, q)=J_{q}(1)
$$

where $J_{q}(t)=\left.\partial_{s} \exp _{p_{s}}\left(t W_{s}(q)\right)\right|_{s=0}$ is the Jacobi field along the geodesic $\gamma_{q}(t)=\exp _{p}\left(t W_{0}(q)\right)$ with

$$
J_{q}(0)=\Xi \quad \text { and } \quad J_{q}^{\prime}(0):=\frac{D J_{q}}{d t}(0)=\left.\frac{D W_{s}(q)}{d s}\right|_{s=0} .
$$

Note that for $q=Y_{p, v^{\varepsilon, p}}(x)$, we have

$$
J_{q}^{\prime}(0)=\left.\frac{D W_{s}(q)}{d s}\right|_{s=0}=d_{p} v^{\varepsilon, p}(x)[\Xi] X+\left.\left(1+v^{\varepsilon, p}(x)\right) x^{i} \frac{D E_{i}^{s}}{d s}\right|_{s=0}=d_{p} v^{\varepsilon, p}(x)[\Xi] X
$$

and thus by (3.20), we get

$$
\left|J_{q}^{\prime}(0)\right| \leq C \varepsilon^{2}|\Xi|_{g} .
$$


Since also $t \mapsto J_{q}(t)$ satisfies an homogenous second order linear differential equation with uniformly bounded coefficients with respect to $\varepsilon$ and $q$, we get for all $q \in Y_{p, v^{\varepsilon, p}}\left(B_{1}\right)$

$$
\left|J_{q}(1)\right|_{\bar{g}} \leq C\left(\left|J_{q}^{\prime}(0)\right|_{g}+\left|J_{q}(0)\right|_{g}\right) \leq C|\Xi|_{g} .
$$

Thanks to [Proposition 3.6, in [10]], we have

$$
\bar{g}\left(J_{q}(1), \gamma_{q}^{\prime}(1)\right)=\bar{g}\left(J_{q}^{\prime}(0), \gamma_{q}^{\prime}(0)\right)+\bar{g}\left(J_{q}(0), \gamma_{q}^{\prime}(0)\right) .
$$

It is plain that at any point $q=Y_{p, v^{\varepsilon, p}}(x) \in \partial B_{1+v^{\varepsilon, p}}^{\bar{g}}(p)$

$$
\bar{g}\left(J_{q}(1), \bar{\nu}(q)\right)=\bar{g}\left(J_{q}(1), \bar{\nu}(q)-\gamma_{q}^{\prime}(1)\right)+\bar{g}\left(J_{q}(1), \gamma_{q}^{\prime}(1)\right)
$$

which implies

$$
\begin{aligned}
\bar{g}\left(J_{q}(1), \bar{\nu}(q)\right)-\bar{g}(\Xi, X) & =\bar{g}\left(J_{q}(1), \bar{\nu}(q)-\gamma_{q}^{\prime}(1)\right)+\bar{g}\left(J_{q}^{\prime}(0), \gamma_{q}^{\prime}(0)\right)+\bar{g}\left(\Xi, \gamma_{q}^{\prime}(0)-X\right) \\
& =\bar{g}\left(J_{q}(1), \bar{\nu}(q)-\gamma_{q}^{\prime}(1)\right)+\bar{g}\left(J_{q}^{\prime}(0),\left(1+v^{\varepsilon, p}\right) X\right)+v^{\varepsilon, p} \bar{g}(\Xi, X),
\end{aligned}
$$

where we have used the fact that $\gamma_{q}^{\prime}(0)=W_{0}(q)=\left(1+v^{\varepsilon, p}(x)\right) X$. We also have (see for instance [37] for the expansion of $-\bar{\nu}(q)$ and recall (2.4))

$$
\left|\bar{\nu}(q)-\gamma_{q}^{\prime}(1)\right|_{\bar{g}}=\left|\bar{\nu}(q)-\left(1+v^{\varepsilon, p}(x)\right) \Upsilon(x)\right|_{\bar{g}} \leq C \varepsilon^{2} .
$$

By using this (3.20), (3.34) and (3.35), we then deduce that, at any point $q=Y_{p, v^{\varepsilon, p}}(x) \in$ $\partial B_{1+v^{\varepsilon, p}}^{\bar{g}}(p)$,

$$
\left|\bar{g}\left(J_{q}(1), \bar{\nu}(q)\right)-\bar{g}(\Xi, X)\right|_{g} \leq C \varepsilon^{2}|\Xi|_{g}
$$

We now recall that

$$
\Phi_{\varepsilon}\left(p_{s}\right):=J\left(\xi\left(s, B_{1+v^{\varepsilon, p}}^{\bar{g}}(p)\right)\right)+\frac{1}{N^{2}}\left|\xi\left(s, B_{1+v^{\varepsilon, p}}^{\bar{g}}(p)\right)\right|_{\bar{g}} .
$$

Assume now that $p$ is a critical point of $\Phi_{\varepsilon}$. Then by Lemma 3.7 and the last equation of (3.32), we have

$$
\begin{aligned}
0=\frac{d}{d s} \Phi_{\varepsilon}\left(p_{s}\right)_{\left.\right|_{s=0}} & =-\int_{\partial B_{1+v^{\varepsilon, p}}^{\bar{g}}(p)}\left[\bar{g}\left(\nabla_{\bar{g}} \bar{\phi}, \bar{\nu}\right)\right]^{2} \bar{g}\left(J_{q}(1), \bar{\nu}\right) d \sigma_{\bar{g}}+\frac{1}{N^{2}} \int_{\partial B_{1+v^{\varepsilon, p}}^{\bar{g}}(p)} \bar{g}\left(J_{q}(1), \bar{\nu}\right) d \sigma_{\bar{g}} \\
& =-\frac{2}{N} \int_{\partial B_{1+v^{\varepsilon, p}}^{\bar{g}}(p)} \bar{g}\left(J_{q}(1), \bar{\nu}\right) d \sigma_{\bar{g}}-\int_{\partial B_{1+v^{\varepsilon}, p}^{\bar{g}}(p)} \bar{g}\left(A^{\varepsilon, p}, \mathcal{V}^{\varepsilon, p}\right)^{2} \bar{g}\left(J_{q}(1), \bar{\nu}\right) d \sigma_{\bar{g}} .
\end{aligned}
$$

Hence

$$
\frac{2}{N} \int_{\partial B_{1+v^{\varepsilon}, p}^{\bar{g}}(p)} \bar{g}\left(A^{\varepsilon, p}, \mathcal{V}^{\varepsilon, p}\right) \bar{g}\left(J_{q}(1), \bar{\nu}\right) d \sigma_{\bar{g}}=-\int_{\partial B_{1+v^{\varepsilon}, p}^{\bar{g}}(p)} \bar{g}\left(A^{\varepsilon, p}, \mathcal{V}^{\varepsilon, p}\right)^{2} \bar{g}\left(J_{q}(1), \bar{\nu}\right) d \sigma_{\bar{g}}
$$

and by (3.35)

$$
\frac{2}{N} \int_{\partial B_{1+v^{\varepsilon}, p}^{\bar{g}}(p)} \bar{g}\left(A^{\varepsilon, p}, \mathcal{V}^{\varepsilon, p}\right) \bar{g}\left(J_{q}(1), \bar{\nu}\right) d \sigma_{\bar{g}} \leq c\|\Xi\|_{\hat{g}} \int_{\partial B_{1+v^{\varepsilon}, p}^{\bar{g}}(p)} \bar{g}\left(A^{\varepsilon, p}, \mathcal{V}^{\varepsilon, p}\right)^{2} d \sigma_{\bar{g}}
$$

for all $\Xi \in T_{p} \mathcal{M}$. By changing variables, using (3.35) and (3.37), we obtain

$$
\frac{2}{N} \int_{\partial B_{1}}\left\langle a^{\varepsilon, p}, x\right\rangle\langle\Xi, X\rangle d \sigma_{\hat{g}}(x) \leq c \varepsilon^{2}\left\|\Xi|| \hat{g}\left|a^{\varepsilon, p}\right|+c\right\| \Xi \|_{\hat{g}} \int_{\partial B_{1}}\left\langle a^{\varepsilon, p}, x\right\rangle^{2} d \sigma_{\hat{g}}(x) .
$$


From Lemma 2.2, we get

$$
\frac{2}{N} \int_{\partial B_{1}}\left\langle a^{\varepsilon, p}, x\right\rangle\langle\Xi, X\rangle d x \leq c \varepsilon^{2}\|\Xi\|_{\hat{g}}\left|a^{\varepsilon, p}\right|+c\|\Xi\|_{\hat{g}} \int_{\partial B_{1}}\left\langle a^{\varepsilon, p}, x\right\rangle^{2} d x .
$$

We now choose $\Xi=\sum_{i=1}^{N} a_{i}^{\varepsilon, p} E_{i} \in T_{p} \mathcal{M}$ and use the fact that $\left|a^{\varepsilon, p}\right| \leq c \varepsilon^{2}$ to get

$$
\frac{1}{2 N} \int_{\partial B_{1}}\left\langle a^{\varepsilon, p}, x\right\rangle^{2} d x \leq C \varepsilon^{2}\left|a^{\varepsilon, p}\right|^{2}\left(1+\left|a^{\varepsilon, p}\right|\right),
$$

for some positive constant $C$ provided $\varepsilon$ is small. We then conclude that

$$
\frac{1}{2 N}\left|a^{\varepsilon, p}\right|^{2} \leq C \varepsilon^{2}\left|a^{\varepsilon, p}\right|^{2}\left(1+\left|a^{\varepsilon, p}\right|\right),
$$

provided $\varepsilon$ is small enough. This shows that $a^{\varepsilon, p}=0$.

\subsection{Expansion of volumes of the perturbed geodesic ball.}

Lemma 3.10. Assume that $v_{0}^{\varepsilon, p}$ is given by Proposition 3.5. Then for all $\varepsilon$ positive small, we have

$$
v_{0}^{\varepsilon, p}=-\frac{S_{g}(p)}{3 N(N+2)} \varepsilon^{2}+O_{p}\left(\varepsilon^{4}\right) .
$$

Proof. Under the hypothesis of Lemma 3.10, we have with Proposition 3.5 that

$$
\hat{g}\left(\nabla_{\hat{g}} \hat{\phi}^{\varepsilon, p}, \hat{\nu}\right)=-\frac{1}{N}-\left\langle a^{\varepsilon, p}, x\right\rangle \text { on } \partial B_{1} .
$$

Using (3.16), we get equivalently

$$
G\left(p, \varepsilon, v_{0}^{\varepsilon, p}, \bar{v}^{\varepsilon, p}\right)+\left\langle a^{\varepsilon, p}, x\right\rangle=0 \text { on } \partial B_{1} .
$$

Equation (3.13) together with the estimate in proposition 3.5 yield

$$
\frac{1}{N} \mathbb{L}\left(\bar{v}^{\varepsilon, p}\right)-\frac{1}{N} v_{0}^{\varepsilon, p}+\left(\partial_{\nu} \psi_{\varepsilon}\right)_{\left.\right|_{\partial B_{1}}}+\left\langle a^{\varepsilon, p}, x\right\rangle+O_{p}\left(\varepsilon^{4}\right)=0,
$$

where $\psi_{\varepsilon}$ is solution of (3.14). Because the integral of the maps $\mathbb{L}\left(\bar{v}^{\varepsilon, p}\right)$ and $\left\langle a^{\varepsilon, p}, x\right\rangle$ over $S^{N-1}$ are equal to 0 , we get integrating (3.38) that

$$
\begin{aligned}
v_{0}^{\varepsilon, p}\left|B_{1}\right| & =\int_{S^{N-1}} \partial_{\nu} \psi_{\varepsilon} \operatorname{dvol}_{S^{N-1}}+O_{p}\left(\varepsilon^{4}\right) \\
& =\int_{B_{1}} \Delta \psi_{\varepsilon}+O_{p}\left(\varepsilon^{4}\right)=\frac{1}{3 N} \sum_{i, j, k=1}^{N} R_{i j i k} \int_{B_{1}} x^{k} x^{j} \varepsilon^{2}+O_{p}\left(\varepsilon^{4}\right),
\end{aligned}
$$

where we have used (3.14), the notation

$$
R_{i j k l}=g\left(R_{p}\left(E_{i}, E_{k}\right) E_{j}, E_{l}\right) \quad \text { and } \quad R_{i k j l, m}=g\left(\nabla_{E_{m}} R_{p}\left(E_{i}, E_{k}\right) E_{j}, E_{l}\right)
$$

and the fact that the integral of a spherical harmonic odd degree over the unit sphere $S^{N-1}$ is equal to 0 . Now, using the identity

$$
\int_{\partial B_{1}} x^{k} x^{l} \operatorname{dvol}_{S^{N-1}}=\left|B_{1}\right| \delta_{k l},
$$


we deduce that

$$
v_{0}^{\varepsilon, p}=-\frac{S_{g}(p)}{3 N(N+2)} \varepsilon^{2}+O_{p}\left(\varepsilon^{4}\right),
$$

Proposition 3.11. Assume that $v_{0}^{\varepsilon, p}, \bar{v}^{\varepsilon, p}$ are as in Proposition 3.5. Then as $\varepsilon \rightarrow 0$, we have

$$
\left|\partial B_{\varepsilon\left(1+v^{\varepsilon, p}\right)}^{g}(p)\right|_{g}=N\left|B_{1}\right| \varepsilon^{N-1}\left(1-\frac{N+4}{6(N+2)} S_{g}(p) \varepsilon^{2}+O_{p}\left(\varepsilon^{4}\right)\right)
$$

and

$$
\left|B_{\varepsilon\left(1+v^{\varepsilon, p}\right)}^{g}(p)\right|_{g}=\left|B_{1}\right| \varepsilon^{N}\left(1-\frac{1}{2(N+2)} S_{g}(p) \varepsilon^{2}+O_{p}\left(\varepsilon^{4}\right)\right) .
$$

Proof. Recall that $\bar{g}=\varepsilon^{-2} g$ and this implies

$$
\left|B_{\varepsilon\left(1+v^{\varepsilon, p}\right)}^{g}(p)\right|_{g}=\varepsilon^{N}\left|B_{1+v^{\varepsilon, p}}^{\bar{g}}(p)\right|_{\bar{g}}=\varepsilon^{N}\left|B_{1}\right|_{\hat{g}}
$$

and

$$
\left|\partial B_{\varepsilon\left(1+v^{\varepsilon, p}\right)}^{g}(p)\right|_{g}=\varepsilon^{N-1}\left|\partial B_{1}\right|_{\hat{g}} .
$$

We get from the expansion in Lemma 2.2 that

$$
\begin{aligned}
\sqrt{|\hat{g}|} & =1+N v_{0}^{\varepsilon, p}+N \chi \bar{v}^{\varepsilon, p}+\langle x, \nabla \rho\rangle+\frac{1}{6} \sum_{k, l, s=1}^{N} R_{s k s l} x^{k} x^{l} \varepsilon^{2} \\
& +\frac{1}{12} \sum_{k, l, s, m=1}^{N} R_{s k s l, m} x^{k} x^{l} x^{m} \varepsilon^{3}+O_{p}\left(\varepsilon^{4}\right),
\end{aligned}
$$

The expansion of $\left|\partial B_{1}\right|_{\hat{g}}$ then follows integrating (3.41) over the unit sphere $S^{N-1}$, where we use the value of $v_{0}^{\varepsilon, p}$ in Lemma 3.10, the identity (3.39) and the fact that, the integral over the unit sphere $S^{N-1}$ of a spherical harmonic of odd degree is equal to 0 and the function $\bar{v}^{\varepsilon, p}$ has mean value equal to 0 . Similarly we get $\left|B_{1}\right|_{\hat{g}}$ by integrating (3.41) over the unit ball $B_{1}$.

\section{Proof of the Theorem 1.1}

In the following result, we characterize critical points of the function $\Phi_{\varepsilon}$ leading to the location of the extremal domains we have constructed in the previous sections. We recall the reduced functional defined in (3.33) by

$$
\Phi_{\varepsilon}(p):=J\left(B_{1+v^{\varepsilon, p}}^{\bar{g}}(p)\right)+\frac{1}{N^{2}}\left|B_{1+v^{\varepsilon, p}}^{\bar{g}}(p)\right|_{\bar{g}} .
$$

Lemma 4.1. As $\varepsilon$ tends to zero, we have

$$
\Phi_{\varepsilon}(p)=\alpha_{N}+\beta_{N} \varepsilon^{2} S_{g}(p)+O_{p}\left(\varepsilon^{4}\right),
$$

where 


$$
\alpha_{N}=\frac{N^{3}(N+2)+\left|B_{1}\right|^{2}}{N^{2}\left|B_{1}\right|} \quad \text { and } \quad \beta_{N}=\frac{N^{2}(N+2)^{3}-(N+4)\left|B_{1}\right|^{2}}{2 N^{2}(N+2)(N+4)} .
$$

In addition $\beta_{N} \neq 0$ for every $N \geq 2$.

Proof. After change of variable, we can write $\Phi_{\varepsilon}$ on the form

$$
\Phi_{\varepsilon}(p):=\frac{1}{\int_{B_{1}} \hat{\phi}^{\varepsilon, p} \operatorname{dvol}_{\hat{g}}}+\frac{1}{N^{2}}\left|B_{1}\right|_{\hat{g}} .
$$

From the estimate of $\bar{v}^{\varepsilon, p}$ in Proposition $3.5, \hat{\phi}^{\varepsilon, p}$ is now written as

$$
\hat{\phi}^{\varepsilon, p}=\phi_{0}-|x|^{2} \frac{v_{0}^{\varepsilon, p}}{N}-\frac{1}{N}|x|^{2} \chi \bar{v}^{\varepsilon, p}+\Psi_{\varepsilon, v^{\varepsilon, p}}+O_{p}\left(\varepsilon^{4}\right),
$$

where $\Psi_{\varepsilon, v^{\varepsilon, p}}$ is given by Lemma 3.2 with $\rho=v_{0}^{\varepsilon, p}+\chi \bar{v}^{\varepsilon, p}$. We integrate the function $\hat{\phi}^{\varepsilon, p}$ over the unit ball $B_{1}$ using the volume element of $\hat{g}$.

We get, using (3.41) and the fact that $\bar{v}^{\varepsilon, p}$ has zero mean value,

$$
\begin{aligned}
\int_{B_{1}} \hat{\phi}^{\varepsilon, p} \operatorname{dvol}_{\hat{g}} & =\left(1+N v_{0}^{\varepsilon, p}\right) \int_{B_{1}} \phi_{0}-\frac{v_{0}^{\varepsilon, p}}{N} \int_{B_{1}}|x|^{2} \\
& +\int_{B_{1}} \Psi_{\varepsilon, v}+\frac{1}{6} \sum_{k, l, s=1}^{N} R_{s k s l} \varepsilon^{2} \int_{B_{1}} x^{k} x^{l} \phi_{0}+O_{p}\left(\varepsilon^{4}\right) .
\end{aligned}
$$

A straightforward computation yields

$$
\int_{B_{1}} \phi_{0}(|x|)=\frac{\left|B_{1}\right|}{N(N+2)}, \quad \int_{B_{1}}|x|^{2}=\frac{N\left|B_{1}\right|}{N+2}
$$

and

$$
\sum_{k, l, s=1}^{N} R_{s k s l} \varepsilon^{2} \int_{B_{1}} x^{k} x^{l} \phi_{0} \mathrm{dvol}_{S^{N-1}}=\frac{-\left|B_{1}\right| S_{g}(p)}{N(N+2)(N+4)} \varepsilon^{2},
$$

In other to compute the integral of $\Psi_{\varepsilon, v^{\varepsilon, p}}$ over $B_{1}$, we use the formula

$$
\int_{\partial B_{1}}\left(\phi_{0} \frac{\partial \Psi_{\varepsilon, v}}{\partial \nu}-\Psi_{\varepsilon, v} \frac{\partial \phi_{0}}{\partial \nu}\right) \operatorname{dvol}_{S^{N-1}}=\int_{B_{1}}\left(\phi_{0} \Delta \Psi_{\varepsilon, v}-\Psi_{\varepsilon, v} \Delta \phi_{0}\right)
$$

Recall that $\frac{\partial \phi_{0}}{\partial \nu}=-\frac{1}{N}, \phi_{0}=0$ on $\partial B_{1}$ and $-\Delta \phi_{0}=1$ in $B_{1}$. Using this, we get from (4.7) that

$$
\begin{aligned}
\int_{B_{1}} \Psi_{\varepsilon, v} & =\frac{1}{N} \int_{\partial B_{1}} \Psi_{\varepsilon, v} \operatorname{dvol}_{S^{N-1}}-\int_{B_{1}} \phi_{0} \Delta \Psi_{\varepsilon, v} \\
& =\frac{\left|B_{1}\right|}{N} v_{0}^{\varepsilon, p}-\frac{1}{3 N} \sum_{i, j, k=1}^{N} R_{i j i k} \varepsilon^{2} \int_{B_{1}} \phi_{0} x^{k} x^{j}+O_{p}\left(\varepsilon^{4}\right) .
\end{aligned}
$$

Therefore

$$
\int_{B_{1}} \Psi_{\varepsilon, p}=\frac{\left|B_{1}\right|}{N} v_{0}^{\varepsilon, p}+\frac{\left|B_{1}\right| S_{g}(p)}{3 N^{2}(N+2)(N+4)} \varepsilon^{2}+O_{p}\left(\varepsilon^{4}\right) .
$$


Replacing (4.8), (4.5) and (4.6) in (4.4), we obtain

$$
\int_{B_{1}} \hat{\phi}^{\varepsilon, p} \operatorname{dvol}_{\hat{g}}=\frac{\left|B_{1}\right|}{N(N+2)}\left(1+(N+2) v_{0}^{\varepsilon, p}-\frac{(N-2) S_{g}(p)}{6 N(N+4)} \varepsilon^{2}+O_{p}\left(\varepsilon^{4}\right)\right) .
$$

One can now consider the value of $v_{0}^{\varepsilon, p}$ to get

$$
\int_{B_{1}} \hat{\phi}^{\varepsilon, p} \operatorname{dvol}_{\hat{g}}=\frac{\left|B_{1}\right|}{N(N+2)}\left(1-\frac{N+2}{2 N(N+4)} S_{g}(p) \varepsilon^{2}+O_{p}\left(\varepsilon^{4}\right)\right) .
$$

That is

$$
\left.\frac{1}{\int_{B_{1+v^{\varepsilon, p}}^{\bar{g}}(p)} \hat{\phi}^{\varepsilon, p} \operatorname{dvol}_{\hat{g}}}=\left(1+\frac{N+2}{2 N(N+4)} S_{g}(p) \varepsilon^{2}+O_{p}\left(\varepsilon^{4}\right)\right)\right) J_{1},
$$

where

$$
J_{1}=\frac{N(N+2)}{\left|B_{1}\right|} .
$$

We now use the expansion of $\left|B_{1}\right|_{\hat{g}}$ in Proposition 3.11 which we plug in (4.2) to get (4.1). Next we prove that $\beta_{N} \neq 0$. Suppose on the contrary that for some integer $N \geq 2$ we have $\beta_{N}=0$. Then

$$
\left|B_{1}\right|^{2}=\frac{N^{2}(N+2)^{3}}{N+4} .
$$

We now recall the volume of the unit ball $\left|B_{1}\right|$ in $\mathbb{R}^{N}$. For $N=2 k$, an even integer, it is given by

$$
\left|B_{1}\right|=\frac{\pi^{k}}{2 k k !}
$$

and for $N=2 k+1$ we have

$$
\left|B_{1}\right|=\frac{2^{2 k+1} \pi^{k} k !}{(2 k+1)(2 k+1) !}
$$

These imply that

$$
\frac{16 k^{2}(k+1)^{3}}{k+2}=\left(\frac{\pi^{k}}{2 k k !}\right)^{2}
$$

and

$$
\frac{(2 k+1)^{2}(2 k+3)^{3}}{2 k+5}=\left(\frac{2^{2 k+1} \pi^{k} k !}{(2 k+1)(2 k+1) !}\right)^{2}
$$

The above equalities contradict the fact that $\pi$ is a transcendental number, see [27]. 
We now complete the proof of Theorem 1.1 by defining

$$
\mathcal{F}(p, \varepsilon):=\frac{1}{\beta_{N}} \frac{\Phi_{\varepsilon}(p)-\alpha_{N}}{\varepsilon^{2}} .
$$

It follows from Lemma 4.1 that

$$
\left\|\mathcal{F}(\cdot, \varepsilon)-S_{g}\right\|_{C^{2, \alpha}(\mathcal{M})} \leq C \varepsilon^{2},
$$

for a positive constant $C$ independent of $\varepsilon$. If $p$ is a critical point of $\mathcal{F}(\cdot, \varepsilon)$ then by Proposition 3.9 , we have

$$
\begin{cases}-\Delta_{\bar{g}} \bar{\phi}^{\varepsilon, p}=1 & \text { in } B_{1+v^{\varepsilon, p}}^{\bar{g}}(p) \\ \bar{\phi}^{\varepsilon, p}=0 & \text { on } \partial B_{1+v^{\varepsilon, p}}^{\bar{g}}(p) \\ \bar{g}\left(\nabla_{\bar{g}} \bar{\phi}^{\varepsilon, p}, \bar{\nu}\right)=-\frac{1}{N} & \text { on } \partial B_{1+v^{\varepsilon, p}}^{\bar{g}}(p) .\end{cases}
$$

Now we recall that $\bar{g}=\varepsilon^{-2} g$ and so we put $u_{\varepsilon}=\varepsilon^{2} \bar{\phi}^{\varepsilon, p}$ and $\Omega_{\varepsilon}=B_{\varepsilon\left(1+v^{\varepsilon, p}\right)}^{g}(p)$. It is also clear from the construction that

$$
\left\|u_{\varepsilon}\right\|_{C^{2}\left(\overline{\Omega_{\varepsilon}}\right)} \leq C
$$

We therefore finish the proof of Theorem 1.1.

\section{LOCAL FOLIATION BY BOUNDARIES OF EXTREMAL DOMAINS}

Let $E_{i}^{t}$ be the parallel transport of $E_{i}$ along the geodesic $\exp _{p_{0}}\left(t E_{i}\right)$ for all $i=1, \ldots, N$. For $\tau \in \mathbb{R}^{N}$, we let $q=\exp _{p_{0}}\left(\tau^{i} E_{i}\right)$ and consider as usual

$$
Y_{q, v}(x):=\exp _{q}^{\bar{g}}\left(\left(1+v_{0}+\chi \bar{v}\right) \sum_{i=1}^{N} x^{i} E_{i}^{\tau^{i}}\right) .
$$

Then there exists $v^{\varepsilon, q}$ such that $B_{1+v^{\varepsilon, q}}^{\bar{g}}(q)=Y_{\varepsilon, v^{\varepsilon, q}}\left(B_{1}\right)$ satisfies (3.32).

Let us now assume that $p_{0}$ is a non-degenerate critical point of the scalar curvature function $S_{g}$. Then by the implicit function theorem, Proposition 3.9 and Lemma 4.1 there exists a regular curve $\tau(\varepsilon) \in \mathbb{R}^{N}$ with $|\tau(\varepsilon)| \leq C \varepsilon^{2}$ and such that

$$
\nabla_{g} \mathcal{F}\left(\varepsilon, q_{\varepsilon}\right)=0,
$$

where $q_{\varepsilon}=\exp _{p_{0}}\left(\sum_{i=1}^{N} \tau^{i}(\varepsilon) E_{i}\right)$. Therefore by Proposition 3.9 and a scaling argument we have a smooth function $\phi^{\varepsilon}=\phi^{\varepsilon, q_{\varepsilon}}$ such that

$$
\begin{cases}-\Delta_{g} \phi^{\varepsilon}=1 & \text { in } B_{\varepsilon\left(1+v^{\varepsilon, q \varepsilon}\right)}^{g}\left(q_{\varepsilon}\right) \\ \phi^{\varepsilon}=0 & \text { on } \partial B_{\varepsilon\left(1+v^{\varepsilon, q_{\varepsilon}}\right)}^{g}\left(q_{\varepsilon}\right) \\ g\left(\nabla_{g} \phi^{\varepsilon}, \nu_{\varepsilon}\right)=-\frac{\varepsilon}{N} & \text { on } \partial B_{\varepsilon\left(1+v^{\varepsilon, q_{\varepsilon}}\right)}^{g}\left(q_{\varepsilon}\right) .\end{cases}
$$

We will prove in our next result that the family of hyper-manifolds

$$
\left(\partial B_{\varepsilon\left(1+v^{\left.\varepsilon, q_{\varepsilon}\right)}\right.}^{g}\left(q_{\varepsilon}\right), \quad \varepsilon \in\left(0, \varepsilon_{0}\right)\right)
$$


constitutes a foliation. This is an immediate consequence of Proposition 5.1 below. The main ingredients of the proof is contained in Ye [47]. However we will write a more applicable result.

Proposition 5.1. Let $p_{0} \in \mathcal{M}$ and $\gamma:\left[0, t_{0}\right] \rightarrow \mathcal{M}$ be a regular curve such that $\gamma(0)=p_{0}$ and $\left|\gamma^{\prime}(0)\right|_{g}=0$. Let $v:\left[0, t_{0}\right] \times S^{N-1} \rightarrow \mathbb{R}$ be a $C^{2}$-function such that $v(0, \cdot)=0$.

Then there exists $t_{1} \in\left(0, t_{0}\right)$ and a $C^{2}$-function $\omega:\left(0, t_{1}\right] \times S^{N-1} \rightarrow \mathbb{R}_{+}^{*}$ such that for all $t \in\left(0, t_{1}\right]$

$\left\{\exp _{\gamma(t)}\left(t(1+v(t, x)) \sum_{i=1}^{N} x^{i} E_{i}^{t}\right): x \in S^{N-1}\right\}=\left\{\exp _{p_{0}}\left(\omega(t, y) \sum_{i=1}^{N} y^{i} E_{i}\right): y \in S^{N-1}\right\}$,

where $E_{i}^{t}$ is the parallel transport to $\gamma(t)$ of $E_{i}$ along the geodesic $s \mapsto \exp _{p_{0}}\left(s E_{i}\right)$.

In addition

$$
\partial_{t} \omega(0, \cdot)=1 .
$$

In particular setting $S_{t\left(1+v^{t}\right)}^{g}(\gamma(t))=\left\{\exp _{\gamma(t)}\left(t(1+v(t, x)) \sum_{i=1}^{N} x^{i} E_{i}^{t}\right): x \in S^{N-1}\right\}$, then the family of perturbed balls $\left(S_{t\left(1+v^{t}\right)}^{g}(\gamma(t)), \quad t \in\left(0, t_{1}\right)\right)$ constitutes a smooth foliation of a neighborhood of $p_{0}$.

Proof. To alleviate the notations, we put $v^{t}=v(t, \cdot)$ and $p_{t}=\gamma(t)$.

Claim: There exists a smooth function $w^{t}: S^{N-1} \rightarrow T_{p_{0}} \mathcal{M}$ such that

$$
S_{t\left(1+v^{t}\right)}^{g}\left(p_{t}\right)=\left\{\exp _{p_{0}} w^{t}(x): x \in S^{N-1}\right\}, \quad \text { and } \quad w^{t}(x)=\sum_{i=1}^{N}\left(t x^{i}+o(t)\right) E_{i} .
$$

Recall that

$$
S_{t\left(1+v^{t}\right)}^{g}\left(p_{t}\right)=\left\{\exp _{p_{t}}\left(t\left(1+v^{t}\right) \sum_{i=1}^{N} x^{i} E_{i}^{t}\right): x \in S^{N-1}\right\} .
$$

Let now consider the (well defined) map $\Psi^{t}:=\exp _{p_{0}}^{-1} \circ \exp _{p_{t}}: T_{p_{t}} \mathcal{M} \longrightarrow T_{p_{0}} \mathcal{M}$ and define

$$
\begin{aligned}
F:\left[0, t_{0}\right) \times S^{N-1} \times T_{p_{0}} \mathcal{M} & \longrightarrow T_{p_{0}} \mathcal{M} \\
(t, x, w) & \longmapsto \Psi^{t}\left(t\left(1+v^{t}(x)\right) \sum_{i=1}^{N} x^{i} E_{i}^{t}\right)-w
\end{aligned}
$$

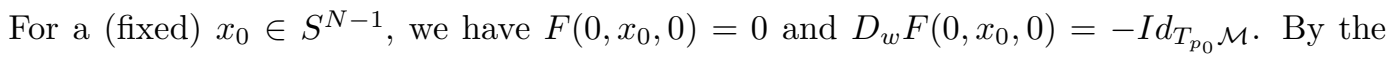
compactness of $S^{N-1}$, the implicit function theorem implies that there exists $t_{1}>0$ such that for all $t \in\left(0, t_{1}\right)$ and for all $x \in S^{N-1}$, there exits a unique $w^{t}(x) \in T_{p_{0}} \mathcal{M}$ such that $F\left(t, x, w^{t}(x)\right)=0$. That is, for all $x \in S^{N-1}$

$$
\exp _{p_{0}}\left(w^{t}(x)\right)=\exp _{p_{t}}\left(t\left(1+v^{t}(x)\right) \sum_{i=1}^{N} x^{i} E_{i}^{t}\right)
$$


In particular, we have $w(0, x)=0$ for all $x$ in $S^{N-1}$. Differentiating (5.2) with respect to $t$, we get

$$
d\left(\exp _{p_{0}}\right)_{0}\left(\left.\frac{\partial w^{t}}{\partial t}\right|_{t=0}\right)=d(\exp )_{p_{0}}\left(\left.\frac{\partial p_{t}}{\partial t}\right|_{t=0}\right)+d\left(\exp _{p}\right)_{0}\left(\sum_{i=1}^{N} x^{i} E_{i}\right) .
$$

By assumption, we have $\left.\frac{\partial p_{t}}{\partial t}\right|_{t=0}=0$ and since

$$
d\left(\exp _{p}\right)_{0}=I d_{T_{p} \mathcal{M}}
$$

we conclude that

$$
{\frac{\partial w^{t}}{\partial t}}_{\left.\right|_{t=0}}=\sum_{i=1}^{N} x^{i} E_{i}
$$

and hence

$$
w^{t}(x)=\sum_{i=1}^{N}\left(t x^{i}+O\left(t^{2}\right)\right) E_{i},
$$

for all $t \in\left(0, t_{1}\right)$ and all $x \in S^{N-1}$ this proves the claim.

Observe that $\left|w^{t}(x)\right|_{g} \neq 0$ for $t>0$ small enough and thus we can consider the map

$$
\alpha:\left(0, t_{1}\right) \times S^{N-1} \rightarrow S^{N-1}
$$

by

It is clear from (5.4) that

$$
\alpha^{i}(t, x)=\frac{1}{\left|w^{t}(x)\right|_{g}} g\left(w^{t}(x), E_{i}\right)
$$

$$
\alpha(t, x)=\frac{x+O(t)}{|x+O(t)|} .
$$

The function $\alpha$ extends smoothly to $t=0$ with $\alpha(0, \cdot)=I d_{S^{N-1}}$ and for $t$ small enough $\alpha(t, \cdot)$ is a diffeomorphism from $S^{N-1}$ into itself. It is plain that for all $x \in S^{N-1}$

$$
w^{t}(x)=\left|w^{t}(x)\right|_{g} \frac{w^{t}(x)}{\left|w^{t}(x)\right|_{g}}
$$

and thus for all $y \in S^{N-1}$

$$
w^{t}\left(\alpha^{-1}(t, y)\right)=\left|w^{t}\left(\alpha^{-1}(t, y)\right)\right|_{g} \sum_{i=1}^{N} y^{i} E_{i} .
$$

This together with (5.2) imply that

$$
S_{t\left(1+v^{t}\right)}^{g}\left(p_{t}\right)=\left\{\exp _{p_{0}}\left(\left|w^{t}\left(\alpha^{-1}(t, y)\right)\right|_{g} \sum_{i=1}^{N} y^{i} E_{i}\right): y \in S^{N-1}\right\} .
$$

We have

$$
\left|\alpha^{-1}(t, y)\right|^{2}=1 \quad \text { for all } t \in\left(0, t_{1}\right) \quad \text { and } y \in S^{N-1}
$$

so that

$$
\left\langle\partial_{t} \alpha^{-1}(t, y), \alpha^{-1}(t, y)\right\rangle=0 .
$$


It then follows that

$$
\begin{aligned}
\partial_{t}\left(\left|w^{t}\left(\alpha^{-1}(t, y)\right)\right|_{g}\right) & =\frac{1}{\left|w^{t}\left(\alpha^{-1}(t, y)\right)\right|_{g}}\left\langle w^{t}\left(\alpha^{-1}(t, y)\right), \partial_{t} w^{t}+\left(d_{x} w^{t}\right)\left(\partial_{t} \alpha^{-1}\right)\right\rangle \\
& =\frac{1}{\left|\alpha^{-1}(t, y)+O(t)\right|}\left\langle\alpha^{-1}(t, y)+O(t), \alpha^{-1}(t, y)+t \partial_{t} \alpha^{-1}+O(t)\right\rangle \\
& =\frac{1}{\left|\alpha^{-1}(t, y)+O(t)\right|}\left\langle\alpha^{-1}(t, y)+O(t), \alpha^{-1}(t, y)+O(t)\right\rangle,
\end{aligned}
$$

where we have used (5.8) to get the last line. Keeping in mind that $\alpha(0,$.$) is the identity$ map, we obtain $\partial_{t}\left(\left|w^{t}\left(\alpha^{-1}(t, y)\right)\right|_{g}\right)_{\left.\right|_{t=0}}=1$. We conclude that map $t \longmapsto\left|w^{t}\left(\alpha^{-1}(t, y)\right)\right|_{g}$ is strictly increasing with respect to $t \in\left(0, t_{1}\right)$ by decreasing $t_{1}>0$ if necessary. Therefore thanks to (5.6), the family $\left\{S_{t\left(1+v^{t}\right)}^{g}\left(p_{t}\right), t \in\left(0, t_{0}\right)\right\}$ constitutes a foliation and also setting $\omega(t, y):=\left|w^{t}\left(\alpha^{-1}(t, y)\right)\right|_{g}$, we finish the proof of the proposition.

Remark 5.2. An application of Proposition 5.1 shows that the critical domains $\Omega_{\varepsilon}$ (in (1.11)) for the first eigenvalue of the Laplace-Beltrami operator constructed by Pacard and Sicbaldi [36] constitutes also a local foliation of a neighborhood of the non-degenerate critical point $p_{0}$ of the scalar curvature. Indeed the improvement of the the distance between the center of their extremal domains and $p_{0}$ was estimated by Sicbaldi and Dilay [8] which is of order $\varepsilon^{2}$.

\section{Proof of Theorem 1.3}

Via the exponential map, we pull back the problem to $\mathbb{R}^{N}$. For this we consider the pull back metric of $g$ under the map $\mathbb{R}^{N} \rightarrow \mathcal{M}, x \mapsto \exp _{p_{0}}\left(\varepsilon \sum_{i=1}^{N} x^{i} E_{i}\right)$, rescaled with the factor $\frac{1}{\varepsilon^{2}}$. Denoting this metric on $B_{1}$ by $g_{\varepsilon}$, we then have, in Euclidean coordinates,

$$
d v_{g_{\varepsilon}}(x):=\sqrt{\left|g_{\varepsilon}\right|}(x)=1-O\left(\varepsilon^{2}\right) .
$$

Call $\Sigma_{\varepsilon}=\partial \Omega_{\varepsilon} \subset \mathbb{R}^{N}$ then it can be easily verified that

$$
\left|\Sigma_{\varepsilon}\right|_{g_{\varepsilon}}=\left|\Sigma_{\varepsilon}\right|\left(1+O\left(\varepsilon^{2}\right)\right) \quad\left|\Omega_{\varepsilon}\right|_{g_{\varepsilon}}=\left|\Omega_{\varepsilon}\right|\left(1+O\left(\varepsilon^{2}\right)\right) .
$$

Integrate the first equality in (1.8) over $\Omega_{\varepsilon}$ to have

$$
\left|\Sigma_{\varepsilon}\right|=\frac{N}{\varepsilon}\left|\Omega_{\varepsilon}\right|(1+o(1)) .
$$

Now since $\Omega_{\varepsilon} \subset \delta_{\varepsilon} B$ by (1.9), we get that

$$
\frac{1}{\varepsilon} \leq\left(\frac{\left|B_{1}\right|}{\left|\Omega_{\varepsilon}\right|}\right)^{\frac{1}{N}}(1+o(1))
$$

and thus

$$
\left|\Sigma_{\varepsilon}\right| \leq(1+o(1)) N\left|\Omega_{\varepsilon}\right|^{\frac{N-1}{N}}\left|B_{1}\right|^{\frac{1}{N}} .
$$

We then conclude by the Euclidean isoperimetric inequality that

$$
c_{N}\left|\Omega_{\varepsilon}\right|^{\frac{N-1}{N}} \leq\left|\Sigma_{\varepsilon}\right| \leq(1+o(1)) c_{N}\left|\Omega_{\varepsilon}\right|^{\frac{N-1}{N}},
$$


where $c_{N}=N|B|^{\frac{1}{N}}$ is the isoperimetric constant of $\mathbb{R}^{N}$. In particular the sets $\Omega_{\varepsilon}$ are almost minimizers for the isoperimetric problem.

Now consider the real numbers $\rho_{\varepsilon} \rightarrow 0$ defined as $\left|\Omega_{\varepsilon}\right|=\left|\rho_{\varepsilon} B_{1}\right|$. Let $\Sigma_{\varepsilon}^{\prime}=\frac{1}{\rho_{\varepsilon}} \Sigma_{\varepsilon}$ and $\Omega_{\varepsilon}^{\prime}=\frac{1}{\rho_{\varepsilon}} \Omega_{\varepsilon}$. Then (6.3) yields

$$
\left|S^{N-1}\right| \leq\left|\Sigma_{\varepsilon}^{\prime}\right| \leq(1+o(1))\left|S^{N-1}\right|, \quad\left|\Omega_{\varepsilon}^{\prime}\right|=\left|B_{1}\right| .
$$

Using this and (6.2) we get

$$
\frac{\rho_{\varepsilon}}{\varepsilon}=1+o(1)
$$

so that

$$
\Omega_{\varepsilon}^{\prime} \subset(1+o(1)) B_{1} .
$$

By compactness $\Sigma_{\varepsilon}^{\prime}$ converges weakly to $b+S^{N-1}$ (see [30]) and also we have that the symmetric distance $\left|\Omega_{\varepsilon}^{\prime} \triangle\left(b+B_{1}\right)\right| \rightarrow 0$ as $\varepsilon \rightarrow 0$, for some point $b \in \mathbb{R}^{N}$. Note that by (6.6), $b=0$. Letting $w_{\varepsilon}(x)=\rho_{\varepsilon}^{-2} u_{\varepsilon}\left(\rho_{\varepsilon} x\right)$, we have

$$
\begin{cases}-\Delta_{\tilde{g}_{\varepsilon}} w_{\varepsilon}=1 & \text { in } \Omega_{\varepsilon}^{\prime} \\ w_{\varepsilon}=0 & \text { on } \partial \Omega_{\varepsilon}^{\prime} \\ \tilde{g}_{\varepsilon}\left(\nabla^{\tilde{g}_{\varepsilon}} w_{\varepsilon}, \nu_{\varepsilon}^{\prime}\right)=-\frac{\varepsilon}{\rho_{\varepsilon} N} & \text { on } \partial \Omega_{\varepsilon}^{\prime},\end{cases}
$$

where $\tilde{g}_{\varepsilon}(x)=g_{\varepsilon}\left(\rho_{\varepsilon} x\right)$. It is also easy to see from (1.10) that

$$
\left\|D^{2} w_{\varepsilon}\right\|_{C\left(\overline{\Omega_{\varepsilon}^{\prime}}\right)} \leq c .
$$

We let $d_{\varepsilon}(x)=\operatorname{dist}\left(\partial \Omega_{\varepsilon}^{\prime}, x\right)$ be the distance function of $\Sigma_{\varepsilon}^{\prime}$. Given $x \in \Omega_{\varepsilon}^{\prime}$ near $\partial \Omega_{\varepsilon}^{\prime}$ then it can be written uniquely as $x=\sigma_{x}-d_{\varepsilon}(x) \nu_{\varepsilon}^{\prime}\left(\sigma_{x}\right)$, where $\sigma_{x}$ is the projection of $x$ on $\Sigma_{\varepsilon}^{\prime}$. This defines coordinates $(t, \sigma) \mapsto x=\sigma-t \nu_{\varepsilon}^{\prime}(\sigma)$. Recall the decomposition of the Laplace-Beltrami operator in the coordinates $(t, \sigma)$ :

$$
\Delta_{\tilde{g}_{\varepsilon}}=\frac{\partial^{2}}{\partial t^{2}}+H_{\varepsilon}^{t} \frac{\partial}{\partial t}+\Delta_{\Sigma_{\varepsilon}^{t}},
$$

where $H_{\varepsilon}^{t}$ is the mean curvature of the hypersurface $\Sigma_{\varepsilon}^{t}=\left\{x \in \Omega_{\varepsilon}^{\prime}: d_{\varepsilon}=t\right\}$ with respect to the metric $\tilde{g}_{\varepsilon}$ and $\Delta_{\Sigma_{\varepsilon}^{t}}$ is the Laplace-Beltrami on $\Sigma_{\varepsilon}^{t}$. We also observe that

$$
\frac{\partial w_{\varepsilon}}{\partial t}=\left|\nabla w_{\varepsilon}\right|_{\tilde{g}_{\varepsilon}}=-\tilde{g}_{\varepsilon}\left(\nabla_{\tilde{g}_{\varepsilon}} w_{\varepsilon}, \nu_{\varepsilon}^{\prime}\right) \quad \text { on } \partial \Omega_{\varepsilon}^{\prime} .
$$

Thanks to (6.8) and the second equation in (1.8), we conclude that

$$
H_{\varepsilon}^{0}=\frac{1-\frac{\partial^{2} w_{\varepsilon}}{\partial t^{2}}}{\frac{\partial w_{\varepsilon}}{\partial t}} \quad \text { on } \partial \Omega_{\varepsilon}^{\prime} .
$$

Therefore

$$
\left|H_{\varepsilon}^{0}\right| \leq \text { Const. } \quad \text { on } \partial \Omega_{\varepsilon}^{\prime} .
$$


Since $\tilde{g}_{\varepsilon}$ is nearly Euclidean, the mean curvature of $\partial \Omega_{\varepsilon}^{\prime}$, with respect to the Euclidean metric, is uniformly bounded with respect to $\varepsilon$. Hence by [35] (see also [23]) the hypersurface $\Sigma_{\varepsilon}^{\prime}$ converges smoothly to $S^{N-1}$ and there exists a function $\tilde{v}^{\varepsilon} \in C^{2, \alpha}\left(S^{N-1}\right)$ with $\left\|\tilde{v}^{\varepsilon}\right\|_{C^{2, \alpha}\left(S^{N-1}\right)} \rightarrow 0$ as $\varepsilon \rightarrow 0$ and such that

$$
\Sigma_{\varepsilon}^{\prime}=\left(1+\tilde{v}^{\varepsilon}\right) S^{N-1} .
$$

We therefore conclude from (6.5) that

$$
\Sigma_{\varepsilon}=\rho_{\varepsilon}\left(1+\tilde{v}^{\varepsilon}\right) S^{N-1}=\varepsilon\left(1+v^{\varepsilon}\right) S^{N-1}
$$

and of course $\left\|v^{\varepsilon}\right\|_{C^{2, \alpha}\left(S^{N-1}\right)} \rightarrow 0$ as $\varepsilon \rightarrow 0$. Hence we get $\Omega_{\varepsilon}=B_{\varepsilon\left(1+v^{\varepsilon}\right)}^{g}\left(p_{0}\right)$ so that the uniqueness of Proposition 3.1 and a scaling argument yield

$$
u_{\varepsilon}=\varepsilon^{2} \bar{\phi}\left(p, \varepsilon, v_{0}^{\varepsilon}, \bar{v}^{\varepsilon}\right) .
$$

Since, by assumption,

$$
\left.\varepsilon^{-1} g\left(\nabla_{g} u_{\varepsilon}, \nu_{\varepsilon}\right)\right|_{\partial B_{\varepsilon\left(1+v^{\varepsilon}\right)}^{g}}=\left.\hat{g}\left(\nabla_{\hat{g}} \hat{u}_{\varepsilon}, \hat{\nu}_{\varepsilon}\right)\right|_{\partial B_{1}}=-\frac{1}{N},
$$

the uniqueness of Proposition 3.5 implies that

$$
\Pi_{1} v^{\varepsilon}=0
$$

provided $\varepsilon$ is small. We now compute the normal derivative of $u_{\varepsilon}$ by using similar arguments as in the proof of Lemma 3.3. It follows that

$$
\left.\varepsilon^{-1} g\left(\nabla_{g} u_{\varepsilon}, \nu_{\varepsilon}\right)\right|_{\partial B_{\varepsilon\left(1+v^{\varepsilon}\right)}^{g}}=-\frac{1}{N}+\frac{1}{N} \mathbb{L}\left(v^{\varepsilon}\right)+\left(\partial_{\nu} \psi_{\varepsilon}\right)_{\left.\right|_{\partial B_{1}}}+\left(\partial_{\nu} \Gamma_{\varepsilon, v^{\varepsilon}}\right)_{\left.\right|_{\partial B_{1}}}+P_{\varepsilon}^{1}\left(v^{\varepsilon}\right),
$$

where $\Gamma_{\varepsilon, v}$ satisfies (3.12) and the function $\psi_{\varepsilon}$ satisfies

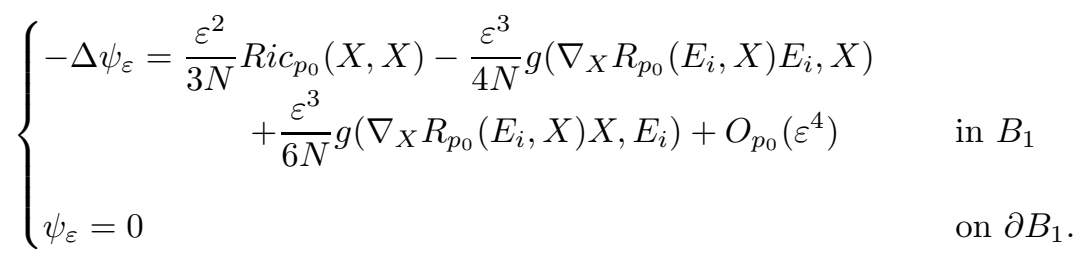

Thanks to (6.9), we have

$$
\frac{1}{N} \mathbb{L}\left(v^{\varepsilon}\right)=-\left(\partial_{\nu} \psi_{\varepsilon}\right)_{\left.\right|_{\partial B_{1}}}+\left(\partial_{\nu} \Gamma_{\varepsilon, v^{\varepsilon}}\right)_{\left.\right|_{\partial B_{1}}}+P_{\varepsilon}^{1}\left(v^{\varepsilon}\right) .
$$

From (3.12), we see immediately from elliptic regularity theory that

$$
\left\|\Gamma_{\varepsilon, v^{\varepsilon}}\right\|_{C^{2, \alpha}\left(S^{N-1}\right)} \leq C \varepsilon^{4}+C \varepsilon^{2}\left\|v^{\varepsilon}\right\|_{C^{2, \alpha}\left(S^{N-1}\right)}+C\left\|v^{\varepsilon}\right\|_{C^{2, \alpha}\left(S^{N-1}\right)}^{2} .
$$

Recalling (6.10), we then apply Proposition 3.4 in (6.12) to have

$$
\left\|v^{\varepsilon}\right\|_{C^{2, \alpha}\left(S^{N-1}\right)} \leq C \varepsilon^{2}+C \varepsilon^{2}\left\|v^{\varepsilon}\right\|_{C^{2, \alpha}\left(S^{N-1}\right)}+C\left\|v^{\varepsilon}\right\|_{C^{2, \alpha}\left(S^{N-1}\right)}^{2} .
$$

This implies that

$$
\left\|v^{\varepsilon}\right\|_{C^{2, \alpha}\left(S^{N-1}\right)} \leq C \varepsilon^{2}
$$

We then conclude that

$$
\mathbb{L}\left(v^{\varepsilon}\right)=-N\left(\partial_{\nu} \psi_{\varepsilon}\right)_{\partial_{\partial B_{1}}}+O\left(\varepsilon^{4}\right)
$$


Now we multiply this equation by $x^{i}$, integrate by parts over $B_{1}$, use (6.11) together with Bianchi's identity to get

$$
\nabla_{g}^{i} S_{g}\left(p_{0}\right)=0
$$

\section{ApPENDix:}

As mentioned in the first section, the torsional rigidity of the $\operatorname{rod} \Omega \times \mathbb{R}$ is proportional to the inverse of

$$
J(\Omega):=\inf \left\{\int_{\Omega}|\nabla u|_{g}^{2} \mathrm{dvol}_{g}: \int_{\Omega} u \operatorname{dvol}_{g}=1, u \in H_{0}^{1}(\Omega)\right\} .
$$

In particular minimizing $\Omega \mapsto J(\Omega)$ is equivalent to maximizing the torsion rigidity and therefore Serrin's result states that balls maximize the torsion rigidity as it can be also derived from the Faber-Krahn inequality.

In this appendix we consider the isochoric profile for the torsion problem defined as

$$
\mathcal{T}_{\mathcal{M}}(v, g):=\inf _{\Omega \subset \mathcal{M},|\Omega|_{g}=v} J(\Omega),
$$

where here and in the following, we assume without further mention that only regular bounded domains $\Omega \subset \mathcal{M}$ are considered. In particular thanks to the Faber-Krahn inequality

$$
\mathcal{T}_{\mathbb{R}^{N}}(v)=J\left(B_{1}\right)\left(\frac{\left|B_{1}\right|}{v}\right)^{-\frac{N+2}{N}} .
$$

Similarly in the space of constant sectional curvatures, balls minimize $J$, see [32]. Isochoric comparison for $\mathcal{T}$ has been studied recently in the papers [21], [46]. Here we deal with local asymptotics of this profile as $v \rightarrow 0$. This also leads to isochoric comparison in terms of scalar curvature.

In the recent years, several works have been devoted to the Taylor expansion of isoperimetric and ischoric profile for some geometric quantities such as the (relative) perimeter functional, Cheeger constants, Dirichlet eigenvalue, second Neuman eigenvalue, etc. We refer the papers $[4-6,11,12,14,15,19,35]$. We should mention that the argument in this section will follow closely Druet [12] where he studied the expansion of the Faber-Krahn profile. The main result of this section is contained in the following

Theorem 7.1. Let $(\mathcal{M}, g)$ be a compact Riemannian manifold of dimension $N \geq 2$. As $v \rightarrow 0$, we have

$$
\mathcal{T}_{\mathcal{M}}(v, g)=\left[1-\frac{N+6}{6 N(N+4)}\left(\frac{v}{\left|B_{1}\right|}\right)^{\frac{2}{N}} \max _{\mathcal{M}} S_{g}+O\left(v^{\frac{3}{N}}\right)\right] \mathcal{T}_{\mathbb{R}^{N}}(v),
$$

where $S_{g}$ is the scalar curvature of $(\mathcal{M}, g)$.

Proof. The first step of the proof is to derive the expansion of $J\left(B_{\varepsilon}^{g}(p)\right)$ as $\varepsilon \rightarrow 0$. Once this is done we then obtain an upper bound for $\mathcal{T}_{\mathcal{M}}(v, g)$ as $v \rightarrow 0$. The second step consists 
in using the asymptotic profile of the isoperimetric profile for the perimeter functional obtained by Druet in [12] together with the Faber Krahn inequality on the space of constant sectional curvatures. This later step follows exactly Druet [11]. Therefore we will only give the proof of the first step.

Claim: As $v \rightarrow 0$, we have

$$
\mathcal{T}_{\mathcal{M}}(v, g) \leq\left[1-\frac{N+6}{6 N(N+4)}\left(\frac{v}{\left|B_{1}\right|}\right)^{\frac{2}{N}} \max _{\mathcal{M}} S_{g}+O\left(v^{\frac{3}{N}}\right)\right] \mathcal{T}_{\mathbb{R}^{N}}(v) .
$$

To see this we determine the Taylor expansion of $J\left(B_{\varepsilon}^{g}(p)\right)$ as $\varepsilon \rightarrow 0$. Recall that $J\left(B_{\varepsilon}^{g}(p)\right)$ is the Dirichlet energy in the ball $B_{\varepsilon}^{g}(p)$ and $u_{\varepsilon}$ the corresponding minimizer, that is

$$
\begin{cases}-\Delta_{g} u_{\varepsilon}=J\left(B_{\varepsilon}^{g}(p)\right) & \text { in } B_{\varepsilon}^{g}(p) \\ u_{\varepsilon}=0 & \text { on } \partial B_{\varepsilon}^{g}(p) \\ \int_{B_{\varepsilon}^{g}(p)} u_{\varepsilon} \operatorname{dvol}_{g}=1 . & \end{cases}
$$

More precisely, we have that

$$
J\left(B_{\varepsilon}^{g}(p)\right) \leq \int_{B_{\varepsilon}^{g}(p)}\left|\nabla_{g} u\right|^{2} \mathrm{dvol}_{g}
$$

for all $u \in H_{0}^{1}\left(B_{\varepsilon}^{g}(p)\right)$ such that $\int_{B_{\varepsilon}^{g}(p)} u_{\varepsilon} \operatorname{dvol}_{g}=1$. Via the exponential map, we pull back the problem to the unit ball $B_{1} \subset \mathbb{R}^{N}$. For this we consider the pull back metric of $g$ under the map $B_{1} \rightarrow \mathcal{M}, x \mapsto \exp _{p}(\varepsilon x)$, rescaled with the factor $\frac{1}{\varepsilon^{2}}$. Denoting this metric on $B_{1}$ by $g_{\varepsilon}$, we then have, in Euclidean coordinates,

$$
\operatorname{dvol}_{g_{\varepsilon}}(x)=\sqrt{\left|g_{\varepsilon}\right|}(x)=1-\frac{\varepsilon^{2}}{6} \operatorname{Ric}_{p}(X, X)+O\left(\varepsilon^{3}\right)
$$

for $x \in \bar{B}_{1}$ by Proposition 2.1. We consider the function $\varphi_{\varepsilon}(x):=\varepsilon^{N} u_{\varepsilon}(\varepsilon x)$ and we recall in (2.2) the expansion of the scaled metric $\tilde{g}_{\varepsilon}(x)=g(\varepsilon x)$ for $x \in B_{1}$. From (7.4), we get

$$
\begin{cases}-\Delta_{g_{\varepsilon}} \varphi_{\varepsilon}=J\left(B_{\varepsilon}^{g}(p)\right) \varepsilon^{N+2} & \text { in } B_{1} \\ \varphi_{\varepsilon}=0 & \text { on } \partial B_{1} \\ \int_{B_{1}} \varphi_{\varepsilon}(x) \operatorname{dvol}_{g_{\varepsilon}}=1 . & \end{cases}
$$

The functions $\varphi_{\varepsilon}$ are positive in $B_{1}$ and equal to 0 on the boundary. Thank to (7.5), we obtain

$$
J\left(B_{\varepsilon}^{g}(p)\right) \varepsilon^{N+2} \leq \int_{B_{1}}\left|\nabla_{g_{\varepsilon}} \varphi_{\varepsilon}\right|^{2} \mathrm{dvol}_{g_{\varepsilon}}
$$

for all $u \in H_{0}^{1}\left(B_{1}\right)$ such that $\int_{B_{1}} u$ dvol $g_{\varepsilon}=1$. Since the metric $g_{\varepsilon} \longrightarrow g_{0}$ as $\varepsilon \longrightarrow 0$ this immediately implies $\limsup _{\varepsilon \rightarrow 0} J_{\varepsilon} \varepsilon^{N+2} \leq J_{1}$, where $J_{1}=J\left(B_{1}\right)$. Using equation (7.7) and regularity results, the sequence $\left(\varphi_{\varepsilon}\right)$ is uniformly bounded in $C^{2}\left(B_{1}\right)$, and we can write $\int_{B_{1}} \varphi_{\varepsilon}=1+O_{p}(\varepsilon)$ and $\int_{B_{1}}\left|\nabla \varphi_{\varepsilon}\right|^{2}=\varepsilon^{N+2} J\left(B_{\varepsilon}^{g}(p)\right)+O_{p}(\varepsilon)$. This implies that 
$\liminf _{\varepsilon \rightarrow 0} \varepsilon^{N+2} J\left(B_{\varepsilon}^{g}(p)\right) \geq J_{1}$. So we have proved that $J\left(B_{\varepsilon}^{g}(p)\right) \varepsilon^{N+2} \longrightarrow J_{1}$ as $\varepsilon \longrightarrow 0$. Since $\left(\varphi_{\varepsilon}\right)$ is uniformly bounded in $C^{2}\left(B_{1}\right)$ and any subsequence has to converge to the (unique) solution of the limit equation $-\Delta \varphi=J_{1}$ in $B_{1}$ with $\int_{B_{1}} \varphi=1$ and $\varphi \geq 0$, we deduce that $\varphi_{\varepsilon} \longrightarrow \varphi$ in $C^{1}\left(\overline{B_{1}}\right)$ as $\varepsilon \longrightarrow 0$. We multiply (7.7) by $\varphi$ and we get after integrating by parts,

$$
J\left(B_{\varepsilon}^{g}(p)\right) \varepsilon^{N+2}=-\int_{B_{1}} \varphi_{\varepsilon} \Delta_{g_{\varepsilon}} \varphi \operatorname{dvol}_{g_{\varepsilon}} .
$$

By Lemma 2.2 we have

$$
\begin{aligned}
\Delta_{g_{\varepsilon}} \varphi & =\Delta \varphi-\frac{1}{3} \sum_{k, l, i, j=1}^{N} R_{i k j l} x^{k} x^{l} \varepsilon^{2} \partial_{i j}^{2} \varphi+\frac{2}{3} \sum_{i, j, k=1}^{N} R_{i j i k} x^{k} \varepsilon^{2} \partial_{j} \varphi+O_{p}\left(\varepsilon^{3}\right) \\
& =-J_{1}-\frac{1}{3} \sum_{k, l, i, j=1}^{N} R_{i k j l} x^{k} x^{l} \varepsilon^{2} \partial_{i j}^{2} \varphi+\frac{2}{3} \sum_{i, j, k=1}^{N} R_{i j i k} x^{k} \varepsilon^{2} \partial_{j} \varphi+O_{p}\left(\varepsilon^{3}\right)
\end{aligned}
$$

and thus

$$
\begin{aligned}
J\left(B_{\varepsilon}^{g}(p)\right) \varepsilon^{N+2}-J_{1} \int_{B_{1}} \varphi_{\varepsilon} \mathrm{dvol}_{g_{\varepsilon}} & =\frac{1}{3} \varepsilon^{2} \sum_{k, l, i, j=1}^{N} R_{i k j l} \int_{B_{1}} x^{k} x^{l} \varphi_{\varepsilon} \partial_{i j}^{2} \varphi \\
& -\frac{2}{3} \varepsilon^{2} \sum_{i, j, k=1}^{N} R_{i j i k} \int_{B_{1}} x^{k} \varphi_{\varepsilon} \partial_{j} \varphi+O_{p}\left(\varepsilon^{3}\right) .
\end{aligned}
$$

Thanks to (7.7) and the convergence of $\varphi_{\varepsilon}$ to $\varphi$, a straightforward computation using also (4.6) yields

$$
\begin{aligned}
\sum_{k, l, i, j=1}^{N} R_{i k j l} \int_{B_{1}} x^{k} x^{l} \varphi \partial_{i j}^{2} \varphi & =-\frac{J_{1}^{2}}{N} \sum_{k, l, i=1}^{N} R_{i k i l} \int_{B_{1}} x^{k} x^{l} \phi_{0} \\
& =\frac{J_{1}}{N(N+4)} S_{g}(p)=\sum_{i, j, k=1}^{N} R_{i j i k} \int_{B_{1}} x^{k} \varphi \partial_{j} \varphi
\end{aligned}
$$

and thus we get

$$
J\left(B_{\varepsilon}^{g}(p)\right) \varepsilon^{N+2}=J_{1}\left(1-\frac{S_{g}(p)}{3 N(N+4)} \varepsilon^{2}+O_{p}\left(\varepsilon^{3}\right)\right),
$$

where $\varphi$ is given by $\varphi=\phi_{0} /\left\|\phi_{0}\right\|_{L^{1}\left(B_{1}\right)}$ and $\phi_{0}$ is the unique solution of (3.2). Next recall the expansion of volume of geodesic balls which can be deduce from (7.6):

$$
\left|B_{\varepsilon}^{g}(p)\right|_{g}=\varepsilon^{N}\left|B_{1}\right|\left(1-\frac{1}{6(N+2)} \varepsilon^{2} S_{g}(p)+O\left(\varepsilon^{3}\right)\right) .
$$

This together with (7.9) implies that

$$
J\left(B_{\varepsilon}^{g}(p)\right)=\left[1-\frac{N+6}{6 N(N+4)}\left(\frac{v}{\left|B_{1}\right|}\right)^{\frac{2}{N}} S_{g}(p)+O\left(v^{\frac{3}{N}}\right)\right] \mathcal{T}_{\mathbb{R}^{N}}(v),
$$

where $v=\left|B_{\varepsilon}^{g}(p)\right|_{g}$. This then proves the claim as $p$ is arbitrary. 
As said above, the reverse inequality of (7.3) follows step-by-step [12] so we skip the details.

\section{REFERENCES}

[1] A. D. Alexandrov, Uniqueness Theorem for surfaces in large I, Vestnik Leningrad Univ. Math. 11 (1956), $5-17$.

[2] A. Ambrosetti and M. Badiale, Variational perturbative methods and bifurcation of bound states from the essential spectrum, Proc. Roy. Soc. Edinburgh Sect. A 128, (1998), 1131-1161.

[3] A. Ambrosetti and A. Malchiodi, Perturbation Methods and Semilinear Elliptic Problems on $R^{n}$. Progress in Mathematics, Birkhäuser Verlag, Basel-Boston-Berlin (2005).

[4] P. Berard and D. Meyer, Inégalités isopérimétriques et applications. Ann. Sci. Éc. Norm. Supér., IV. Sér. 15, (1982) 513-541.

[5] V. Bayle and C. Rosales, Some isoperimetric comparison theorems for convex bodies in Riemannian manifolds, Indiana Univ. Math. J. 54 (2005), 1371-1394.

[6] I. Chavel, Eigenvalues in Riemannian geometry, Academic Press, 1984.

[7] M. Choulli and A. Henrot, Use of the Domain Derivative to Prove Symmetry Results in Partial Differential Equations. Mathematische Nachrichten, Volume 192, Issue 1, pages 91-103, 1998.

[8] E. Daley and P. Sicbaldi Extremal domains for the first eigenvalue of the Laplace Beltrami operator in a general compact riemannian manifold arXiv:1302.4221v1.

[9] M. del Pino, F. Pacard and J. Wei, Serrin's Overdetermined problem and constant mean curvature surfaces. Preprint. http://arxiv.org/abs/1310.4528v1

[10] M. do Carmo, Riemannian Geometry. Boston: Birkhauser. (1992).

[11] O. Druet, Asymptotic expansion of the Faber-Krahn profile of a compact Riemannian manifold. C. R. Math. Acad. Sci. Paris 346 (2008), no. 21-22, 1163-1167.

[12] O. Druet, Sharp local isoperimetric inequalities involving the scalar curvature, Proceedings of the American Mathematical Society, 130, 8, 2351-2361, (2002).

[13] A. El Soufi and S. Ilias, Domain deformations and eigenvalues of the Dirichlet Laplacian in Riemannian manifold, Illinois Journal of Mathematics 51 (2007) 645-666.

[14] M. M. Fall, Area-minimizing sets in Riemannian manifolds with boundary constrained to small volume. Pacific J. Math. 244 (2010), no. 2, 235-260.

[15] M. M. Fall, Some local eigenvalue estimates involving curvatures. Calc. Var. Partial Differential Equations 36 (2009), no. 3, 437-451.

[16] M. M. Fall and C. Mercuri, Minimal disc-type surfaces embedded in a perturbed cylinder. Differential Integral Equations 22 (2009), no. 11-12, 1115-1124.

[17] M. M. Fall and C. Mercui, Foliations of small tubes in Riemannian manifolds by capillary minimal discs, Nonlinear Analysis TMA. (12) Vol. 70 (2009) 4422-4440.

[18] M. M. Fall and F. Mahmoudi, Hyper-surfaces with free boundary and large constant mean curvature: concentration along sub-manifolds. Ann. Scuola Norm. Sup. Pisa Cl. Sci. (5) Vol. VII (2008), 1-40.

[19] M. M. Fall and T. Weth, Sharp local upper bound of the first non-zero Neumann eigenvalue in Riemannian manifolds. To appear in Calc. Var. Partial Differential Equations. http://arxiv.org/abs/1110.4770.

[20] A. Farina and B. Kawohl, Remarks on overdetermined boundary value problem Calc. Var. Partial Differential Equations 31 (2008), n0 3. 351-357.

[21] S. Gallot, A. Loi and C. Lucio, Maximizing torsional rigidity on Riemannian manifolds. http://arxiv.org/abs/1309.7796.

[22] D. Henry, Perturbation of the Boundary in Boundary-Value Problems of Partial Differential Equations. Cambridge University Press. 2005. 
[23] D. L. Johnson D.L. and F. Morgan, Some sharp isoperimetric theorems for riemannian manifolds. Indiana Univ. Math. J., (2000) 49 (2).

[24] N. Kapouleas, Compact constant mean curvature surfaces in Euclidean three-space. J. Differ. Geom. 33, No.3, (1991), 683-715.

[25] L. D. Landau and E.M. Lifshitz, Theory of Elasticity, Course of Theoretical Physics 7, Pergamon Press, London, 1959.

[26] P. Laurin, Concentration of CMC surfaces in a Riemannian manifold, Int. Math. Res. Not. IMRN 2012, no. 24, 5585-5649.

[27] F. Lindemann, Über die Zahl $\pi$. Math. Ann. 20, 213-225, 1882.

[28] F. Mahmoudi, R. Mazzeo and F. Pacard, Constant mean curvature hypersurfaces condensing along a submanifold, Geom. funct. anal. Vol. 16 (2006) 924-958.

[29] R. Mazzeo, F. Pacard, Foliations by constant mean curvature tubes, Comm. Anal. Geom. 13 (2005), no. $4,633-670$.

[30] F. Maggi, Sets of finite perimeter and geometric variational problems: an introduction to Geometric Measure Theory, Cambridge Studies in Advanced Mathematics no. 135, Cambridge University Press, 2012.

[31] A. Malchiodi and M. Montenegro, Boundary concentration phenomena for a singularly perturbed elliptic problem, Comm. Pure Appl. Math. 55 (2002), no. 12, 1507-1568.

[32] P. Mcdonald, Isoperimetric Conditions, Poisson Problems, and Diffusions in Riemannian Manifolds. Potential Analysis 16: 115-138, 2002.

[33] A. M. Micheletti, A. Pistoia, Generic properties of critical points of the scalar curvature for a Riemannian manifold, Proc. Amer. Math. Soc. 138 (2010), no. 9, 3277-3284.

[34] F. Morabito and P. Sicbaldi, Delauney type domains for an overdetermined elliptic problem in $S^{n} \times \mathbb{R}$ and $\mathbb{H} \times \mathbb{R}$. Preprint. http://arxiv.org/abs/1305.6516

[35] S. Nardulli, Régularité des solutions du probléme isopérimétrique proches de variétés lisses. Preprint Université de Paris Sud, Octobre 2006.

[36] F. Pacard and P. Sicbaldi, Extremal domains for the first eigenv alue of the Laplace-Beltrami operator. Ann. Inst. Fourier (Grenoble) 59 (2009), no. 2, 515-542.

[37] F. Pacard, X. Xu, Constant mean curvature sphere in Riemannian manifolds. Manuscripta Math. 128 (2009), no. 3, 275-295.

[38] S. Raulot and A. Savo, On the spectrum of the Dirichlet-to-Neumann operator acting on forms of a Euclidean domain. J. Geom. Phys. 77 (2014) 1-12.

[39] F. Schlenk and P. Sicbaldi, Bifurcating extremal domains for the first eigenvalue of the Laplacian. Adv. Math. 229 (2012) 602-632.

[40] R. Schoen and S.T. Yau, Lectures on Differential Geometry, International Press (1994).

[41] J. Serrin, A Symmetry Theorem in Potential Theory. Arch. Rational Mech. Anal. 43 (1971), 304-318.

[42] P. Sicbaldi, New extremal domains for the first eigenvalue of the Laplacian in flat tori. Calc. Var. (2010) 37:329-344.

[43] T. Sun, A note on constant geodesic curvature curves on surfaces. Ann. Inst. H. Poincaré Anal. Non Linéaire, 26(5) :1569-1584, 2009.

[44] H. F. Weinberger, An isoperimetric inequality for the $N$-dimensional free membrane problem. J. Rational Mech. Anal. 5 (1956), 633-636.

[45] T.J. Willmore, Riemannian Geometry, Oxford Univ. Press. NY. (1993).

[46] J. Xiao, Isoperimetry for semilinear torsion problems in Riemannian two-manifolds. Advances in Mathematics 229 (2012) 2379-2404.

[47] R. Ye Foliation by constant mean curvature spheres . Pacific J. Math. 147 (1991), no. 2, 381-396. 
African Institute for Mathematical Sciences (A.I.M.S.) of Senegal KM 2, Route de Joal, B.P. 1418 Mbour, SÉnÉgal

E-mail address: mouhamed.m.fall@aims-senegal.org, ignace.a.minlend@aims-senegal.org. 